\title{
Semi-annual, annual and Universal Time variations in the magnetosphere and in geomagnetic activity: 1. Geomagnetic data
}

\author{
Mike Lockwood $^{1, *}$, Mathew J. Owens ${ }^{1}$, Luke A. Barnard ${ }^{1}$, Carl Haines ${ }^{1}$, Chris J. Scott ${ }^{1}$, \\ Kathryn A. McWilliams ${ }^{2}$, and John C. Coxon ${ }^{3}$ \\ ${ }^{1}$ Department of Meteorology, University of Reading, Earley Gate, PO Box 243, Reading RG6 6BB, UK \\ ${ }^{2}$ Institute of Space and Atmospheric Studies, University of Saskatchewan, Saskatoon, SK S7N 5E2, Canada \\ ${ }^{3}$ School of Physics and Astronomy, University of Southampton, Southampton SO17 1BJ, UK
}

Received 13 September 2019 / Accepted 18 May 2020

\begin{abstract}
We study the semi-annual variation in geomagnetic activity, as detected in the geomagnetic indices $a m, a a_{\mathrm{H}}, A L, D s t$ and the four $a \sigma$ indices derived for 6-hour MLT sectors (around noon, dawn, dusk and midnight). For each we compare the amplitude of the semi-annual variation, as a fraction of the overall mean, to that of the corresponding variation in power input to the magnetosphere, $P_{\alpha}$, estimated from interplanetary observations. We demonstrate that the semi-annual variation is amplified in the geomagnetic data compared to that in $P_{\alpha}$, by a factor that is different for each index. The largest amplification is for the Dst index (factor 10) and the smallest is for the $a \sigma$ index for the noon MLT sector $(a \sigma$-noon, factor $\approx 1.1$ ). By sorting the data by the prevailing polarity of the $Y$-component (dawn-dusk) of the Interplanetary Magnetic Field (IMF) in the Geocentric Solar Equatorial (GSEQ) reference frame, we demonstrate that the Russell-McPherron (R-M) effect, in which a small southward IMF component in GSEQ is converted into geoeffective field by Earth's dipole tilt, is a key factor for the semi-annual variations in both $P_{\alpha}$ and geomagnetic indices. However, the variability in the southward component in the IMF in the GSEQ frame causes more variability in power input to the magnetosphere $P_{\alpha}$ than does the R-M effect, by a factor of more than two. We show that for increasingly large geomagnetic disturbances, $P_{\alpha}$ delivered by events of large southward field in GSEQ (known to often be associated with coronal mass ejections) becomes the dominant driver and the R-M effect declines in importance and often acts to reduce geoeffectiveness for the most southward IMF in GSEQ: the semi-annual variation in large storms therefore suggests either preconditioning of the magnetosphere by average conditions or an additional effect at the equinoxes. We confirm that the very large R-M effect in the Dst index is because of a large effect at small and moderate activity levels and not in large storms. We discuss the implications of the observed "equinoctial" time-of-year $(F)$ - Universal Time $(U T)$ pattern of geomagnetic response, the waveform and phase of the semi-annual variations, the differences between the responses at the June and December solstices and the ratio of the amplitudes of the March and September equinox peaks. We also confirm that the UT variation in geomagnetic activity is a genuine global response. Later papers will analyse the origins and implications of the effects described.
\end{abstract}

\section{Introduction}

The semi-annual variation in geomagnetic activity has long been recognized (Broun, 1848; Sabine, 1852; Cortie, 1912; Chapman \& Bartels, 1940; McIntosh, 1959; Russell \& McPherron, 1973; Cliver et al., 2002; Le Mouël et al., 2004) and gives peaks at times close to both equinoxes in both the average levels of geomagnetic activity and the occurrence frequency of large geomagnetic disturbances. Figure 1 stresses that

\footnotetext{
*Corresponding author: m. 1ockwood@reading. ac.uk
}

this is a highly persistent phenomenon by plotting the variation in each year of the "homogeneous" version of the $a a$ geomagnetic index, $a a_{\mathrm{H}}$. This index is available from 1868 to the present day (Lockwood et al., 2018a, b; see URL given in the Acknowledgements and data sources section) and is based on the same observations as the classic aa index (Mayaud, 1971, 1972, 1980): it has been homogenised because it contains allowance for the secular variation in the intrinsic geomagnetic field, employs revised inter-calibrations of the magnetometer stations as a function of time-of-year $(F)$, and deploys a model of the response of a station at a given location, $F$ and Universal 


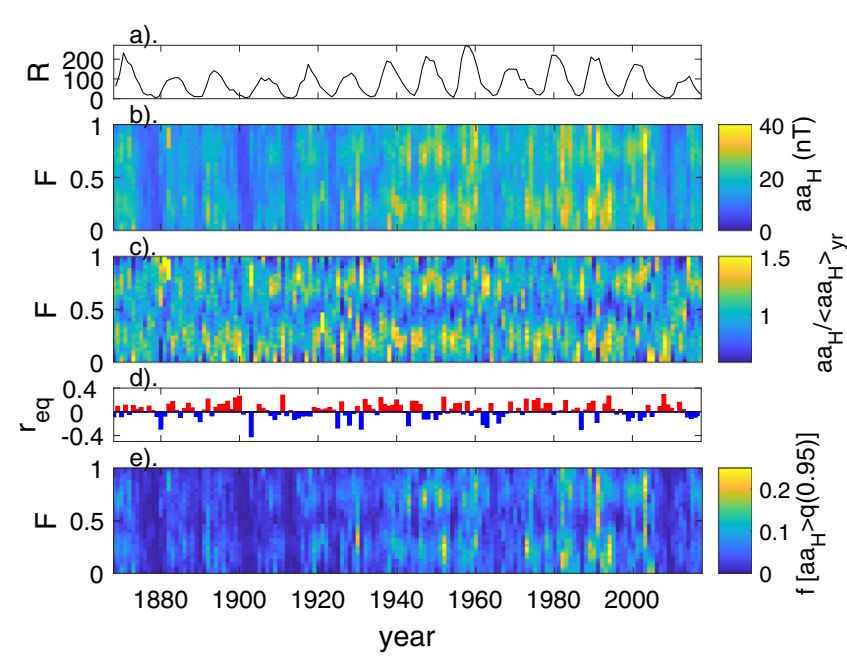

Figure 1. The semi-annual variation in the $a a_{\mathrm{H}}$ index, available since 1868. (a) Annual means of the international sunspot number, $R$; (b) mean values of $a a_{\mathrm{H}}$ in 30 equal-sized bins of time-of-year $F$ as a function of $F$ and year; (c) the mean $a a_{\mathrm{H}}$ values normalized by dividing by the annual mean, $\left\langle a a_{\mathrm{H}}\right\rangle_{\mathrm{yr}}$; (d) a comparison of the means of $a a_{\mathrm{H}}$ in quarter-year intervals around the March and September equinoxes, $\left\langle a a_{\mathrm{H}}\right\rangle_{\mathrm{me}}$ and $\left\langle a a_{\mathrm{H}}\right\rangle_{\mathrm{se}}$ respectively, where $r_{\mathrm{eq}}=\left\{\left\langle a a_{\mathrm{H}}\right\rangle_{\mathrm{me}}-\right.$ $\left.\left\langle a a_{\mathrm{H}}\right\rangle_{\mathrm{se}}\right\} /\left\{\left\langle a a_{\mathrm{H}}\right\rangle_{\mathrm{me}}+\left\langle a a_{\mathrm{H}}\right\rangle_{\mathrm{se}}\right\}$ and (e) the occurrence frequency of 3-hourly $a a_{\mathrm{H}}$ values exceeding its 95 percentile, $q(0.95)=56 \mathrm{nT}$, $f\left[a a_{\mathrm{H}}>q(0.95)\right]$.

Time (UT). The model allows for the effects of solar zenith angle on ionospheric conductivity and of the variation in the proximity of the station to the midnight sector auroral oval (Lockwood et al., 2018a, b). Tests show that the behaviour of $a a_{\mathrm{H}}$, despite being compiled from just two stations, reproduces the am index (compiled from a global network of 25 stations in both hemispheres) well and that $a a_{\mathrm{H}}$ has a much more uniform $F-U T$ response pattern than the original $a a$. index (Lockwood et al., 2019d).

Figure $1 \mathrm{~b}$ shows mean values of $a a_{\mathrm{H}}$ in 30 equal-sized bins of time of year, $F$, for each year, giving 98 3-hourly $a a_{\mathrm{H}}$ samples in each averaging bin. Note that we employ fraction of a year $(F)$ throughout this paper because day-of-year is different for leap years and non-leap years and month number (or decimal month number) does not give intervals of constant duration. The semi-annual variation in $\left\langle a a_{\mathrm{H}}\right\rangle$ is clearly seen with peaks, broadly speaking, around $F=0.22$ (March equinoxes) and $F=0.73$ (September equinoxes), the magnitudes of which rise and fall with the sunspot number, $R$ (shown in Fig. 1a). Figure 1c shows the bin averages, as a ratio the average for the year $\left\langle a a_{\mathrm{H}}\right\rangle_{\mathrm{yr}}$, and reveals that the semi-annual variation is usually still present during solar minimum years, although sometimes weakened. Only for a very small number of years can no semi-annual variation be detected in Figure 1c, as for example during the very deep minima between cycles 23 and 24 (around 2009). Figure 1e shows the occurrence of large $a a_{\mathrm{H}}$ values, by colour-contouring the frequency $f\left[a a_{\mathrm{H}}>\right.$ $q(0.95)]$ with which the 3-hourly $a a_{\mathrm{H}}$ index exceeds its overall 95-percentile value $q(0.95)=56 \mathrm{nT}$. This also shows a strong semi-annual variation that varies in amplitude with $R$. Figure 1d compares the relative amplitude of the two equinox peaks by plotting the asymmetry factor,

$$
r_{\mathrm{eq}}=\left\{\left\langle a a_{\mathrm{H}}\right\rangle_{\mathrm{me}}-\left\langle a a_{\mathrm{H}}\right\rangle_{\mathrm{se}}\right\} /\left\{\left\langle a a_{\mathrm{H}}\right\rangle_{\mathrm{me}}+\left\langle a a_{\mathrm{H}}\right\rangle_{\mathrm{se}}\right\}
$$

where $\left\langle a a_{\mathrm{H}}\right\rangle_{\text {me }}$ and $\left\langle a a_{\mathrm{H}}\right\rangle_{\mathrm{se}}$ are the means of $a a_{\mathrm{H}}$ in quarteryear intervals around the March and September equinoxes, respectively. It can be seen that, on balance, the March equinox tends to show the larger of the two peaks (years with red bars), although the opposite is often true, as it has tended to be in recent years. There is no persistent relationship between the 22-year Hale solar cycle and the variation in $r_{\text {eq }}$. From the lack of any clear pattern to the difference between the two peaks and from its great year-to-year variability, it seems most likely that it is largely a matter of chance as to at which of the two equinoxes in a year the Sun happens to send out more and larger Earth-directed transient disturbances.

The explanations for the semi-annual variation fall into three classes that all arise from the geometrical considerations associated Earth's orbit around the Sun, combined (in two of the cases, at least) with the effects of Earth's rotation due to the offset of Earth's geomagnetic and rotational axes. These are called the "Russell-McPherron" (R-M), the "equinoctial" and the "axial" effects. The R-M and equinoctial effects combine Earth's orbital and rotational variations which means that the semi-annual variation (period 0.5 year) is intrinsically linked with Universal Time (UT) variations (period $24 \mathrm{~h}$ ). Furthermore, the fact that the offset of the rotational and geomagnetic poles is greater in the southern hemisphere than in the northern means that both the semi-annual and $U T$ variations should also be linked with an annual variation (period 1 year). This north-south asymmetry in the geomagnetic field should also mean that the $U T$ variations do not average out over a full year (as they often do in the symmetric case) leaving a net dependence of the magnetosphere on $U T$ (period one day). The axial mechanism is different because it depends only on the heliographic latitude of Earth and so gives no $U T$ variation.

As described in the three following subsections, the "Russell-McPherron" (R-M) F-UT pattern (Fig. 2e) arises from considering the changes in the angle between the GSM (Geocentric Solar Magnetospheric) and GSEQ (Geocentric Solar Equatorial) reference frames (Russell \& McPherron, 1973); the equinoctial F-UT pattern (Fig. 3e) arises from considering the tilt angle of Earth's magnetic axis towards or away from the Sun (Bartels, 1925; McIntosh, 1959) and the axial pattern (Fig. 4f) arises from the variation in Earth's heliographic latitude (Cortie, 1912). All three predict peaks in geomagnetic activity near the equinoxes (but on somewhat different dates and with different $U T$ dependencies).

\subsection{The Russell-McPherron effect}

The concept of the Russell-McPherron (R-M) effect is that the near-Earth heliospheric field lies predominantly in the solar equatorial $(X Y)$ plane of the Geocentric Solar Equatorial frame (GSEQ: the geocentric equivalent of the solar RTN frame) whereas geomagnetic activity responds to southward-pointing field in the Geocentric Solar Magnetospheric (GSM) frame. The angle of rotation between these two frames, $\beta_{\mathrm{GSEQ}}$, modulates the solar wind power that enters the magnetosphere and so can introduce biases into averages of geomagnetic activity. The $X_{\mathrm{GSEQ}}$ axis points from the centre of the Earth to the centre of the Sun, the $Y_{\mathrm{GSEQ}}$ axis is parallel to the solar equatorial plane and the $Z_{\mathrm{GSEQ}}$ axis makes up the right-hand 


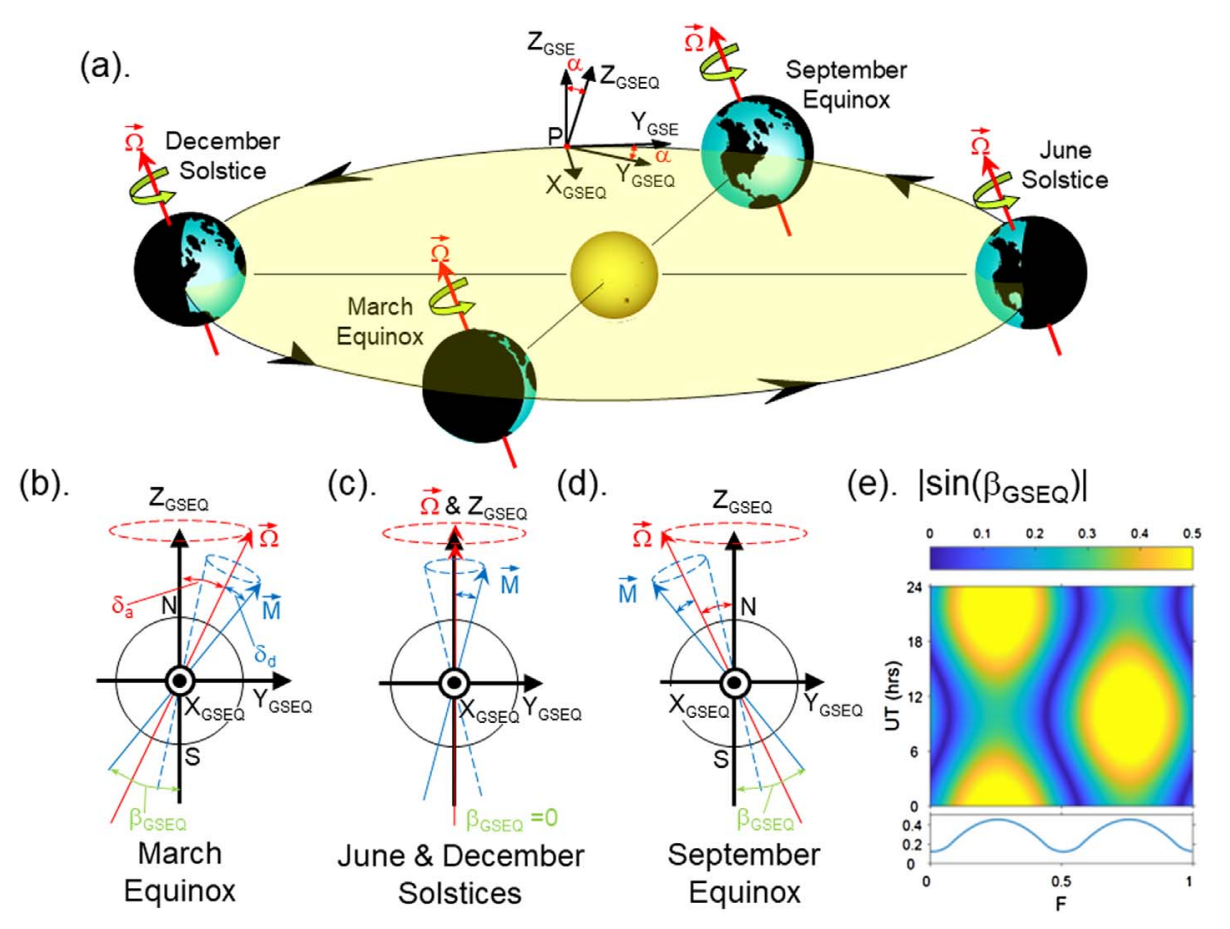

Figure 2. Schematic of the Russell-McPherron (R-M) effect. Part (a) illustrates Earth in its orbit at the June and December solstices and the March and September equinoxes, showing the orientation of Earth's rotational axis $\vec{\Omega}$ in red and defining the ecliptic plane (shaded yellow) and the GSE reference frame. Also shown is the GSEQ reference frame (defined in Fig. 4); (b), (c) and (d) are views looking toward Earth from the Sun (i.e., in the $-X_{\mathrm{GSEQ}}$ direction) and show the $Z_{\mathrm{GSEQ}}$ and $Y_{\mathrm{GSEQ}}$ axes and the projections of $\vec{\Omega}$ and Earth's magnetic axis $\vec{M}$ (in blue): $\vec{\Omega}$ precesses around the $Z_{\mathrm{GSEQ}}$ axis once per year and $\vec{M}$ precesses around $\vec{\Omega}$ every $24 \mathrm{~h}$. The cone angles of these annual and daily precessions are $\delta_{\mathrm{a}}=23.5^{\circ}$ and $\delta_{\mathrm{a}} \approx 9.6^{\circ}$, respectively. (Note that $\delta_{\mathrm{a}}$ of $9.6^{\circ}$ is an approximate figure because Earth's field in not a geocentric dipole; for example, in 2007 the geomagnetic pole was $6.05^{\circ}$ from the rotation pole in the Northern hemisphere but $25.55^{\circ}$ from it in the Southern hemisphere; (b) is the view for the March equinox, (c) for the June and December solstices and (d) is for the September equinox. The GSM (Geocentric Solar Magnetospheric) reference frame is, like GSE and GSEQ, a right handed set and GSEQ, GSE and GSM share the same $X$ axis, but the $Z_{\mathrm{GSM}}$ and $Y_{\mathrm{GSM}}$ axes are rotated through an angle $\beta_{\mathrm{GSEQ}}$ (that varies with Universal Time, UT, and time-of-year, $F$ ) such that $Z_{\mathrm{GSM}}$ is aligned with the projection of $\vec{M}$ onto the $Z-Y$ plane. The idea of the R-M effect is that geomagnetic activity is driven by a coupling function that depends of the southward interplanetary magnetic field (IMF) in the GSM fame, for example, the "half-wave rectified" southward component $B_{\mathrm{S}}$ in the GSM frame (where $B_{\mathrm{S}}=-\left[B_{Z}\right]_{G S M}$ for $\left[B_{Z}\right]_{G S M}<0$ and $B_{\mathrm{S}}=0$ for $\left[B_{Z}\right]_{G S M} \geq 0$ ) but the dominant IMF reaching Earth lies in the GSEQ $X-Y$ plane (so $\left[B_{\mathrm{z}}\right]_{\mathrm{GSEQ}}=0$ ). The resulting $F-U T$ pattern of $B_{\mathrm{S}}$ for $\left|\left[B_{Y}\right]_{\mathrm{GSEQ}}\right|=1 \mathrm{nT}$ is set by $\sin \left(\beta_{\mathrm{GSEQ}}\right)$ and is $\operatorname{shown}$ in the upper panel of part (e) and daily means as a function of $F$ are given in the lower panel. The $F$ variation (currently, 2019) due to R-M peaks around 4 April $(F=0.257)$ and October $7(F=0.769)$ whereas the equinoxes are March $20(F=0.216)$ and September $22(F=0.726)$.

set. Note that in the heliospheric Radial, Tangential and Normal $(R T N)$ coordinates $R$ is radially away from the Sun (and so is anti-parallel to $X_{\mathrm{GSEQ}}$ ), $T$ is normal to the plane formed by the $R$ axis and the Sun's spin vector, positive in the direction of planetary motion (taking the solar equatorial plane to be normal to the spin axis means that $T$ is anti-parallel to $Y_{\mathrm{GSEQ}}$ ), by making up the right hand set, $N$ is parallel to $Z_{\mathrm{GSEQ}}$. GSEQ is similar to, but not the same as, the Geocentric Solar Ecliptic (GSE) frame which uses the same $X$ axis as GSEQ but the $Y_{\mathrm{GSE}}$ axis lies in the ecliptic plane in which Earth orbits (and so $Z_{\mathrm{GSE}}$ is the northward normal to the ecliptic plane). The GSM frame also uses the same $X$ axis but the $Z$ and $Y$ axes are rotated such that $Z_{\mathrm{GSM}}$ is aligned with the projection of the Earth's magnetic axis, $\vec{M}$, onto the $Y Z$ plane (a plane that is common to GSM, GSE and GSEQ frames). The rotation angle between the GSM and GSE frames is $\beta_{\mathrm{GSE}}$ and between the GSM and GSEQ frame is $\beta_{\mathrm{GSEQ}}$. To illustrate the R-M effect, Figure $2 \mathrm{a}$ shows Earth and its rotational axis at four points in its orbit: at the two solstices and the two equinoxes. Figures $2 \mathrm{~b}-2 \mathrm{~d}$ show how the magnetic axis of the Earth $\vec{M}$ appears at certain times when viewed from the Sun: the GSM $Z$ axis is aligned with the projection of $\vec{M}$ onto the $Y Z$ plane and so these figures demonstrate how the rotation of the GSM frame relative to the GSEQ will convert $\left[B_{Y}\right]_{\mathrm{GSEQ}}$ (of the required polarity) into southward $\left[B_{Z}\right]_{\mathrm{GSM}}$. This is significant because the average orientation of the interplanetary magnetic field (IMF) is in the solar equatorial plane, giving zero $\left[B_{Z}\right]_{\mathrm{GSEQ}}$ and large $\left[B_{Y}\right]_{\mathrm{GSEQ}}$, and because it is southward IMF in the GSM frame (i.e., $\left[B_{Z}\right]_{\mathrm{GSM}}<0$ ) that drives geomagnetic activity. Because the rotation angle between the GSE and GSEQ frame $\alpha\left(=\beta_{\mathrm{GSE}}-\beta_{\mathrm{GSEQ}}\right)$ is relatively small, $-7.25^{\circ} \leq \alpha \leq+7.25^{\circ}$, the differences between the IMF components in the GSE and GSEQ frames are generally small. Note that Figure 2 is a revised version of Figure 1 of Lockwood et al. (2016) but uses GSEQ rather than GSE 


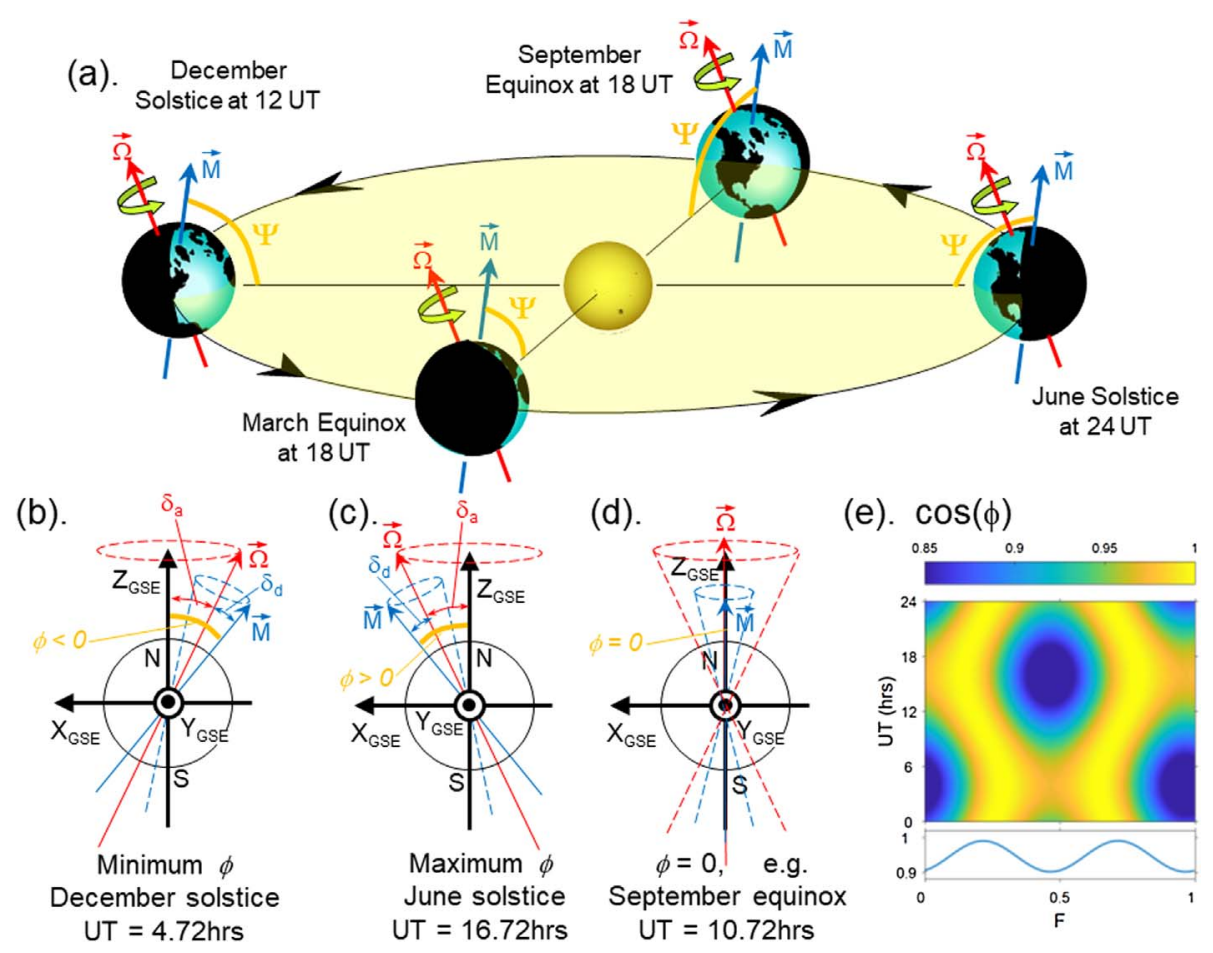

Figure 3. Schematic illustrating the equinoctial effect. Part (a) is the same as in Figure 2, but includes the magnetic $\vec{M}$ axis at selected UTs and the angle $\Psi$ that it makes with the $X_{\mathrm{GSE}}$ axis. Parts (b), (c) and (d) are views of Earth from the dusk side (i.e., in the $-Y_{\mathrm{GSE}}$ direction) and show the $X_{\mathrm{GSE}}$ and $Z_{\mathrm{GSE}}$ axes and the projection of the angle $\Psi$ into this plane, called the dipole tilt angle $\phi$ that varies between a maximum of $\delta_{\mathrm{a}}+\delta_{\mathrm{d}}=+33.1^{\circ}$ in Northern hemisphere summer at $4.72 \mathrm{UT}$ (part b) and a minimum of $-\left(\delta_{\mathrm{a}}+\delta_{\mathrm{d}}\right)=-33.1^{\circ}$ in Southern hemisphere summer at $16.72 \mathrm{UT}$ (part c). There are a variety of $U T$ and $F$ combinations that give $\phi=0$, and one is illustrated in part d. The equinoctial effect applies to anything that varies with $\phi$ or $\cos (\phi)$ and gives the characteristic pattern $F$-UT pattern and $F$ variation of daily means shown in part e. The peaks of the equinoctial pattern are currently on 21 March $(F=0.219)$ and 19 September $(F=0.719)$.

because GSEQ was used by Russell \& McPherron (1973) in formulating the R-M paradigm; note also that the former version contained an error in the sense of $Y_{\mathrm{GSE}}$.

We here use data on the near-Earth IMF in the GSE and GSM frames obtained from the Omni-2 dataset, compiled and maintained by the Space Physics Data Facility at NASA/ Goddard Space Flight Center. We then use the angle $\alpha$ to transform the GSE data into the GSEQ frame. Around the September equinox it is $\left[B_{Y}\right]_{\mathrm{GSEQ}}>0$ that gives southward $\left[B_{Z}\right]_{\mathrm{GSM}}$ through this R-M effect (Fig. 2d); conversely around the March equinox it is $\left[B_{Y}\right]_{\mathrm{GSEQ}}<0$ that gives southward $\left[B_{Z}\right]_{\mathrm{GSM}}$ (Fig. 2b). A key additional element in the R-M effect is nonlinearity in the response of geomagnetic activity to IMF $\left[B_{Z}\right]_{\mathrm{GS}}$, such that northward IMF has much smaller (or zero) effect. This is expected because geomagnetic activity is driven by the effect of magnetic reconnection in the magnetopause (at latitudes between the magnetic cusps) which generates open flux and so facilitates the entry of solar wind mass, energy and momentum into the magnetosphere. This means that the northward $\left[B_{Z}\right]_{\mathrm{GSM}}$ caused by $\left[B_{Y}\right]_{\mathrm{GSEQ}}<0$ at the September equinox and by $\left[B_{Y}\right]_{\mathrm{GSEQ}}>0$ at the March equinox (the "non-favourable" $\left[B_{Y}\right]_{\mathrm{GSEQ}}$ polarities) does not cancel out the effect of the southward $\left[B_{Z}\right]_{G S M}$ generated by the opposite polarity of $\left[B_{Y}\right]_{\mathrm{GSEQ}}$ (the "favourable" polarities). Hence there is a net rise in average activity at both equinoxes, even though favourable and unfavourable $\left[B_{Y}\right]_{\mathrm{GSEQ}}$ occur with very similar distributions. In the original paper (Russell \& McPherron, 1973), this was investigated using an IMF in its average orientation which is in the solar equatorial plane (i.e., $\left[B_{Z}\right]_{\mathrm{GSEO}}=0$ ) and assuming a "half-wave-rectified" solar wind-magnetosphere coupling function (meaning there is no response at all for northward IMF) but, in practice, a lesser non-linearity with the IMF $\left[B_{Z}\right]_{G S M}$ component would still cause the R-M effect. Figure $2 \mathrm{e}$ shows the resulting $F$-UT pattern for the half-wave rectified southward IMF in the GSM frame, $B_{\mathrm{S}}$, defined below in equation (4).

There is a well-known secondary effect that works in concert with the R-M effect and that is the "Rosenberg-Coleman" (R-C) effect, namely that the polarity of the near-Earth $\left[B_{X}\right]_{\mathrm{GSEQ}}$ IMF component further away from the solar equator tends to reflect the polarity of the open flux in the polar coronal hole in that hemisphere (Rosenberg \& Coleman, 1969). Because the coronal holes have a clear single polarity around sunspot minimum, this has most effect at these times but no net effect about a year after sunspot maximum when the solar polar fields reverse and both polarities of open solar flux are found in both hemispheres with roughly equal fluxes. This influences the R-M effect because the polarity of $\left[B_{Y}\right]_{\mathrm{GSEQ}}$ is anti-correlated with that $\left[B_{x}\right]_{\mathrm{GSEQ}}$ for the "gardenhose" orientation of the IMF, which applies for $75 \%$ of the time on average (Lockwood et al., 2019e). This means that the R-C effect influences the relative occurrence of the two polarities of IMF $\left[B_{Y}\right]_{\mathrm{GSEQ}}$ and so 


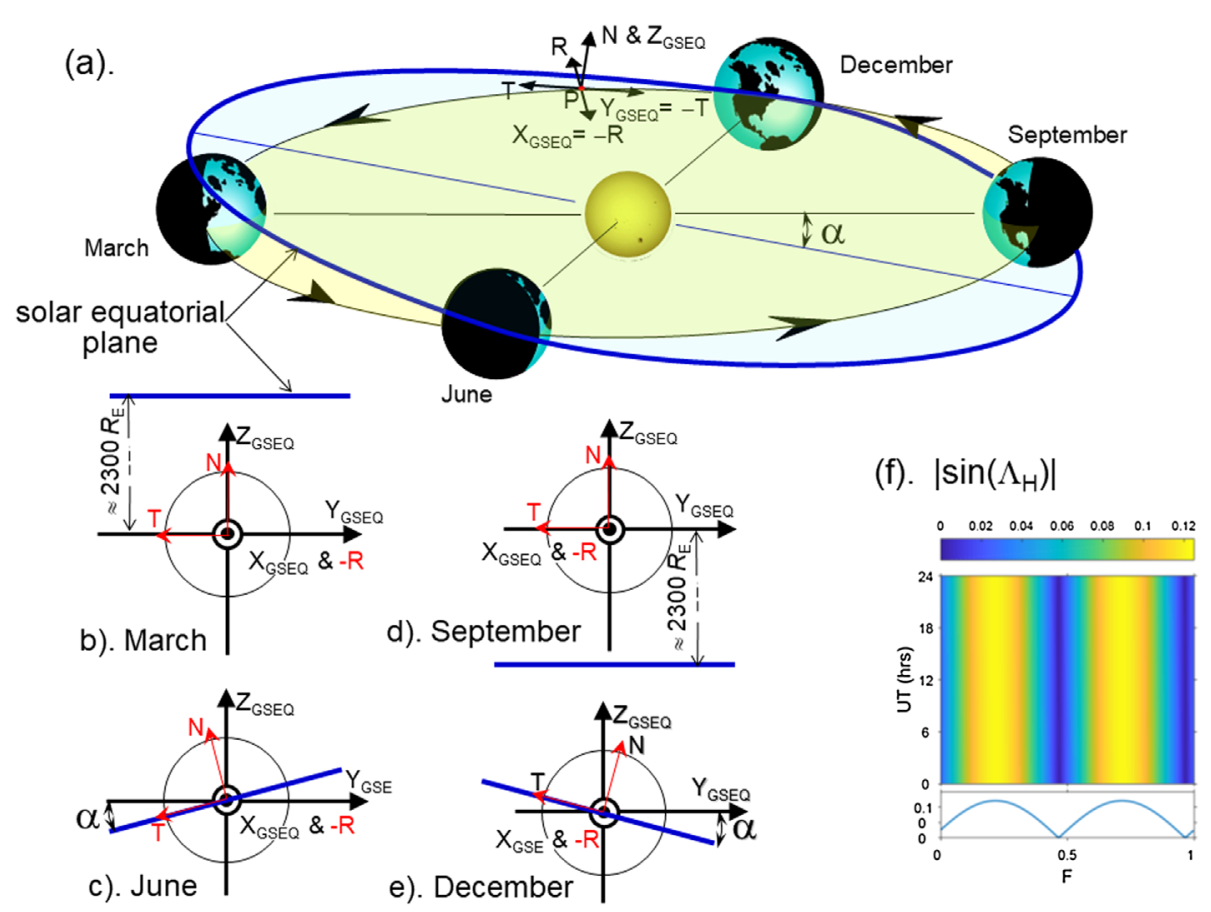

Figure 4. Schematic illustration of the axial effect. Part (a) shows the ecliptic plane as in Figure 2, but added is the Sun's equatorial plane which is inclined at an angle $\alpha=7.25^{\circ}$ to the ecliptic, as shown by the blue circle that is shaded pale blue. This has two effects: firstly, Earth has an annual variation in its heliographic latitude which reaches maximum values in the solar southern and northern hemisphere on dates that happen to be close to the equinoxes, namely $5 \operatorname{March}(F=0.175)$ and 8 September $(F=0.689)$. Secondly there is an annual variation in the relationship between the heliocentric $R T N$ frame, on which the inner heliosphere is structured, and the GSE frame. Parts (b)-(d) show Earth and the heliographic equator looking from the Sun at four times of year (i.e., viewing in the $-X_{\mathrm{GSE}}$ direction). The axial pattern, shown in part $\mathrm{f}$, depends on $F$ but has no $U T$ variation. The GSEQ frame is also defined in the figure: the $X_{\mathrm{GSEQ}}$ axis points from the Earth to the Sun and so is the same as $X_{\mathrm{GSE}}$ a $X_{\mathrm{GSM}}$ axes. Whereas the $Y_{\mathrm{GSE}}$ axis lies in the ecliptic plane, $Y_{\mathrm{GSEQ}}$ is parallel to the Sun's equatorial plane which is inclined to the ecliptic. $Z_{\mathrm{GSEQ}}$ makes up the right hand set and has a component that is positive in a northward direction. Note that since $X_{\mathrm{GSEQ}}$ lies is in the ecliptic plane it is not generally in the Sun's equatorial plane, and $Z_{\mathrm{GSEQ}}$ will not generally be parallel to the Sun's axis of rotation. In term of RTN heliographic coordinates, $X_{\mathrm{GSEQ}}=-R, Y_{\mathrm{GSEQ}}=-T$ and $Z_{\mathrm{GSEQ}}=N$.

can change the net effect of the R-M mechanism at a given time. This is one reason why in this and subsequent papers we frequently perform our analysis separately for the two polarities of IMF $\left[B_{Y}\right]_{\mathrm{GSEQ}}$; the point being that then, although the R-C effect can change the relative number of samples available for analysis of the two $\left[B_{Y}\right]_{\mathrm{GSEQ}}$ polarities, it has no influence on the average R-M effect, as quantified by the extent to which one $\left[B_{Y}\right]_{\mathrm{GSEQ}}$ polarity is converted into $\left[B_{z}\right]_{\mathrm{GSM}}$. Note also that if the combined R-C/R-M effect were a major factor we should see a 22-year Hale cycle effect of the polarity of the solar polar field in which equinox is favoured, particularly at sunspot minima. Figure 1 shows that since 1868, at the eight sunspot minima at the end of even-numbered sunspot cycles we see one with dominant $r_{\text {eq }}<0$, two with dominant $r_{\text {eq }}>0$ and five with a mixture of both; for the seven minima at the end of odd-numbered sunspot cycles we see three with dominant $r_{\text {eq }}<0$, one with dominant $r_{\text {eq }}>0$ and three with a mixture of both. Hence the Hale cycle pattern expected for the combined $\mathrm{R}-\mathrm{C} / \mathrm{R}-\mathrm{M}$ effect around sunspot minimum is not detected.

Figure 3 of O'Brien \& McPherron (2002) and Figure 2d of Lockwood et al. (2016) demonstrate that the R-M F-UT pattern is indeed seen in the average, half-wave-rectified southward component of the IMF in the GSM frame $\left[B_{Z}\right]_{\mathrm{GSM}}$. Furthermore, Lockwood et al. (2016) show that larger disturbances in the Dst index near the March/September equinoxes occur when the prior IMF $\left[B_{Y}\right]_{\mathrm{GSE}}$ has been predominantly negative/ positive, respectively and, as noted by Zhao \& Zong (2012), this is a unique signature of the R-M effect. However, there is a puzzle here because Figure 5 of Lockwood et al. (2016) shows that large negative $\left[B_{Z}\right]_{\mathrm{GSM}}$ is predominantly caused by large negative $\left[B_{Z}\right]_{\mathrm{GSE}}$ and that the role of the GSE to GSM conversion on $\left[B_{Z}\right]_{\mathrm{GSM}}$ is relatively minor. In other words, strongly-geoeffective IMF is mainly caused by large transient events in the solar wind (in particular Coronal Mass Ejections, CMEs) that generate large out-of-ecliptic southward field $\left(\left[B_{Z}\right]_{\mathrm{GSE}} \ll 0\right)$ and the R-M effect on its own is restricted to generating relatively minor enhancements. This generates a paradox at the heart of the R-M mechanism that has not generally been recognised and certainly has not been resolved.

A more recognised, but equally puzzling, feature is that the characteristic F-UT pattern of the R-M effect, shown in Figure $2 \mathrm{e}$, is not observed in geomagnetic disturbance indices. The most revealing index in this respect is $a m$, because it has by far the most uniform response in $F$ and $U T$ (Lockwood et al., 2019d). If an index is compiled from an uneven distribution of magnetometer stations in the two hemispheres then seasonal effects, such as in ionospheric conductivity, will give it a spurious additional variation in $F$ and if it has an uneven 
distribution of stations in longitude (in either or both hemispheres) it will give a spurious additional variation in UT. The am index (Mayaud, 1980) achieves the most uniform $F-U T$ response of all geomagnetic indices by using two longitudinal rings of stations at sub-auroral latitudes (close to a corrected geomagnetic latitude of $50^{\circ}$ ) with 15 stations in the northern hemisphere and 10 in the south. These are grouped into longitude sectors, with five such groups in the Northern hemisphere, and four in the Southern. The data from stations in each group are averaged together: to compensate for the lower number of stations and groups in the southern hemisphere (largely caused by the lack of stations in the Pacific Ocean) and differences in station separations, longitudinal-extent weighting factors are used. The weighted means are combined into northern and southern hemisphere indices, an and as, and $a m=(a n+a s) / 2$. A map of the am station locations is given in Figure 1b of Lockwood et al. (2019d) or can be viewed on the ISGI site, along with a discussion of the grouping procedure and its weighting factors, at http://isgi.unistra.fr/Documents/ am_LWFs_example.pdf. Lockwood et al. (2019d) have simulated the am response to solar forcing using a model of how each station responds as a function of its co-ordinates, $F$ and $U T$, based on the work of Finch (2008): the station responses are then combined using exactly the same procedure as is used to generate the index from observations (further details are given in Sect. 5 below). The results show that am has a very even $F-U T$ response with the largest fluctuations at low geomagnetic activity levels and almost no variation at high activity levels. Specifically, observed am values range between 0 and $619 \mathrm{nT}$ and at $a m<10 \mathrm{nT}$ (which comprises $33 \%$ of the data) the response is constant to within $\pm 2 \%$ and at $a m>30 \mathrm{nT}$ (which is the largest $20 \%$ of the data) it is constant to within $\pm 0.5 \%$. This is considerably better than for any other index. The am index is available for 1959 onward.

\subsection{The Equinoctial Effect and the role of solar wind dynamic pressure}

Instead of revealing the $F-U T$ pattern expected of the R-M effect, the am index shows striking similarities to the equinoctial pattern shown in Figure 3e (de La Sayette \& Berthelier, 1996; Chambodut et al., 2013), although it is not an exact match (Cliver et al., 2000). In addition, Chambodut et al. (2013) have shown that the four $a \sigma$ indices (which are $a m$ indices are generated using data restricted to four 6-hour ranges of Magnetic Local Time (MLT) around noon, dusk, midnight and dawn) all display the equinoctial $F$-UT response pattern but it is most well defined in the midnight sector. The equinoctial element indicates that the tilt of the Earth's magnetic axis $\vec{M}$ towards or away from the Sun has an influence, introducing differences between the two solstices and between 4 UT and 16 UT which are not predicted by the R-M effect (O'Brien \& McPherron, 2002). Figure 3 illustrates the origins of this dipole tilt effect in the same format as Figure 2, except that parts (b), (c) and (d) the relevant view of Earth is from the dusk side. There have been a large number of explanations proposed for this observed equinoctial pattern. Boller \& Stolov (1970) proposed that the angle $\psi$ between $\vec{M}$ the $X_{\mathrm{GSE}}$ axis (shown in Fig. 3a) causes a modulation of the stability of the flanks of the magnetopause to the Kelvin-Helmholtz instability. Svalgaard (1977) suggested that the tilted dipole presents a stronger magnetic field to the solar wind, thereby increasing the magnetopause standoff distance and enlarging the magnetospheric cavity; however, Olson (1969) showed that this idea does not work because the magnetopause stand-off distance varies by less than $3 \%$ over the full range of tilt angles and that the cross-tail dimension changed by less than $1 \%$. Another postulate is that tilt-induced changes in the ionospheric conductivity within the nightside auroral oval have an effect and that the electrojet currents are weaker when conductivities caused by solar EUV are low in midnight-sector auroral ovals of both hemispheres (Lyatsky et al., 2001; Newell et al., 2002). Other proposals invoke tilt influences on the dayside magnetopause reconnection voltage (Crooker \& Siscoe, 1986; Russell et al., 2003) or the effect of tilt on the proximity of the ring current and auroral electrojet (Alexeev et al., 1996) or tilt effects on the stability of the cross-tail current sheet through its curvature (Kivelson \& Hughes, 1990; Danilov et al., 2013; Kubyshkina et al., 2015). All of these effects have the potential to reproduce the equinoctial pattern, but which, if any, are effective remains a matter of debate.

Finch et al. (2008) used a global network of geomagnetic stations to show that the equinoctial behaviour originates during substorm expansion phases and in the substorm current wedge and is not a feature of dayside currents and flows during the substorm growth phase. The results of Finch et al. (2008) therefore strongly support the explanations of the equinoctial effect invoking nightside magnetospheric or ionospheric effects and eliminate those that postulate modulation of the magnetopause reconnection voltage. Lockwood (2013) has pointed out that indices influenced by the substorm current wedge also depend on the solar wind dynamic pressure $p_{\mathrm{SW}}\left(=m_{\mathrm{SW}} N_{\mathrm{SW}} V_{\mathrm{SW}}{ }^{2}\right.$, where $m_{\mathrm{SW}}$ is the mean ion mass, $N_{\mathrm{SW}}$ the number density and $V_{\mathrm{SW}}$ the speed of the solar wind) because it compresses the near-Earth geomagnetic tail and so modulates the near-Earth cross-tail current there for a given open magnetic flux content in the tail (further down the tail lobe magnetic pressure balance is opposed only by the static pressure of interplanetary space meaning that the addition of open flux causes the tail to flare with no increase in lobe field strength nor cross-tail current). This effect was demonstrated directly by Karlsson et al. (2000) who showed that decreases in $p_{\text {Sw }}$ reduced magnetospheric energy content and so caused quenching of any substorm expansion that had recently begun. Many papers have also found the converse effect in which increases in $p_{\mathrm{SW}}$ can trigger onsets of full substorm expansion phases (Schieldge \& Siscoe, 1970; Kokubun et al. 1977; Yue et al., 2010). Caan et al. (1973) showed that the magnetic energy density in the near-Earth tail lobes was increased by both the solar wind dynamic pressure $p_{\mathrm{SW}}$ and by prior southward-pointing IMF. Finch et al. (2008) showed that a $V_{\mathrm{SW}}{ }^{2}$ and $p_{\mathrm{SW}}$ dependence was present in the equinoctial pattern response but not in the directly-driven dayside response. As discussed by Lockwood et al. (2018a, b) and (2019d), mid-latitude range indices (Menvielle \& Berthelier, 1991) respond primarily to the substorm current wedge and so show the effect of $p_{\mathrm{SW}}$. The potential effects of $p_{\mathrm{SW}}$ will be discussed further in later papers.

\subsection{The axial effect}

The idea behind axial effect is illustrated in Figure 4. It arises because the solar equatorial plane (normal to the solar rotation axis) is inclined at an angle $\alpha \approx 7.25^{\circ}$ with respect to 
the ecliptic, such that Earth makes a maximum southward deviation from the solar equator on March 6th and a maximum deviation to the north on September 7th. Earth being at slightly higher heliographic latitude $\left|\Lambda_{\mathrm{H}}\right|$ near the equinoxes increases the probability of it leaving the streamer belt and encountering the fast solar wind (Hundhausen et al., 1971), especially at solar minimum (Whang et al., 2005; McComas et al., 2008; Ebert et al., 2009). An additional effect, also seen near sunspot minimum (when the solar field is more dipolar in form), is the Rosenberg-Coleman (R-C) effect whereby the polarity of $\left[B_{X}\right]_{\text {GSEQ }}$ at Earth reflects that of the polar coronal hole in the same hemisphere to a larger extent at large $\left|\Lambda_{\mathrm{H}}\right|$. As discussed in the last section, this gives biases in $\left[B_{Z}\right]_{\mathrm{GSM}}$ via the R-M effect because $\left[B_{X}\right]_{\mathrm{GSEQ}}$ and $\left[B_{Y}\right]_{\mathrm{GSEQ}}$ are, on average, of opposite sign and related by the Parker spiral orientation. These biases depend on the solar polar field polarity and so change in polarity at successive minima with the 22-year Hale cycle.

For a pure axial mechanism, geomagnetic activity varies with the heliographic latitude $\Lambda_{H}$ (which varies between $-\alpha$ and $+\alpha$ ) and there is no effect of Earth's rotation and so no $U T$ variation. However, note that the combined R-C/R-M effect described above would show a $U T$ variation introduced by the R-M effect. The axial effect appears to be present in the Dst index (see Plate 2 of Cliver et al., 2000 and Fig. $2 \mathrm{f}$ of Lockwood et al., 2016: in the latter case, Dst has been corrected for the longitudinal inhomogeneity in the ring of equatorial stations using the procedure of Takalo \& Mursula, 2001). However, Lockwood et al. (2016) point out that Dst is almost certainly not responding to the variation in Earth's heliographic latitude, rather the long duration of the energy input to the magnetosphere that drives large Dst responses (storms) smooths out the $U T$ variations, giving an axial-like behaviour, as demonstrated in Figure 5i of Lockwood et al. (2016).

\subsection{Comparing the R-M, equinoctial and axial effects}

Figure 5 a plots the variations over a year of the key angles involved in the R-M, equinoctial and axial effects and Figure 5b plots the variations in relevant factors that depend on those angles. The values shown are daily means and so the UT effect is averaged out. The blue line in Figure 5a is the heliographic latitude, $\Lambda_{\mathrm{H}}$. The axial effect is based on the idea that power available for input to the magnetosphere increases with distance away from the solar equator so, if that variation were linear, the variation with time of year $F$ would depend on $\left|\sin \left(\Lambda_{\mathrm{H}}\right)\right|$, shown in blue in Figure 5b. The near-equinox peaks in this factor are shown by vertical blue lines. The dipole tilt angle $\phi$ is shown in mauve and the factor $\cos (\phi)$ gives an equinoctial $F-U T$ pattern. This factor gives peaks (vertical mauve lines) that are later than the peaks for the axial effect by about 16 days for the March equinox $(\Delta F=0.044)$ and by about 10 days for the September equinox $(\Delta F=0.030)$. The relevant angle for the R-M effect is the GSEQ to GSM rotation angle $\beta_{\mathrm{GSEQ}}$, shown in black. For a coupling function that depends on a half-wave-rectified $\left[B_{Z}\right]_{\mathrm{GSM}}$ and an IMF with a $Y$ component in the GSEQ frame of amplitude $\left|\left[B_{Y}\right]_{\mathrm{GSEQ}}\right|=1$, the relevant factor from Figure 2 would be $\left|\sin \left(\beta_{\mathrm{GSEQ}}\right)\right|$. This gives peaks that are later than for the equinoctial effect (vertical black lines) by about 14 and 18 days for the March and September equinoxes, respectively $(\Delta F=0.038$ and $\Delta F=0.050)$. The green line shows the variation expected for unit IMF in the $Y$ direction of the GSE frame, the relevant angle

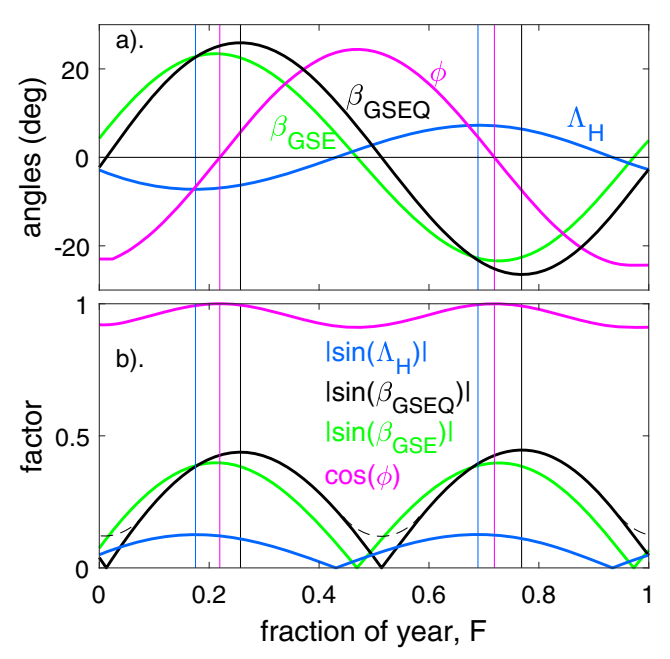

Figure 5. Predictions of the variations with fraction of year, $F$, for the various mechanisms, (a) shows the annual variations of the various angles involved: (blue) the heliographic latitude of Earth, $\Lambda_{\mathrm{H}}$; (mauve) the dipole tilt, $\phi$; (green) the angle $\beta_{\mathrm{GSE}}$ between the $Z$ axes in the GSM and GSE frames of reference; (black) the angle $\beta_{\mathrm{GSEQ}}$ between the $Z$ axes in the GSM and GSEQ frames of reference. The values shown are averages over daily intervals. (b) Factors based on these daily-mean angles: (blue) $\left|\sin \left(\Lambda_{\mathrm{H}}\right)\right|$, the axial effect for an increase in solar wind power with distance away from the solar equator; (mauve) $\cos (\phi)$, a factor that displays the equinoctial effect; (green) $\left|\sin \left(\beta_{\mathrm{GSE}}\right)\right|$, the IMF $\left[B_{Z}\right]_{\mathrm{GSM}}$ component (in $\mathrm{nT}$ ) for an IMF $B=\left[B_{Y}\right]_{\mathrm{GSE}}=1 \mathrm{nT}$ and a half-wave rectification coupling function; (black) $\left|\sin \left(\beta_{\mathrm{GSEQ}}\right)\right|$, the IMF $\left[B_{Z}\right]_{\mathrm{GSM}}$ component (in $\mathrm{nT}$ ) for an IMF $B=\left[B_{Y}\right]_{\mathrm{GSEQ}}=1 \mathrm{nT}$ and a half-wave rectification coupling function. Note the difference between the black solid line which is for daily means of $\beta_{\mathrm{GSEQ}},\left|\sin \left(\left\langle\beta_{\mathrm{GSEQ}}\right\rangle\right)\right|$, and the black dashed line which is for daily means of $\sin \left(\beta_{\mathrm{GSEQ}}\right),\left|\left\langle\sin \left(\beta_{\mathrm{GSEQ}}\right)\right\rangle\right|$ : the averaging of the UT variation means these are not the same on days when higher resolution $\beta_{\mathrm{GSEQ}}$ values (on minute or hour timesacles) are a mixture of both polarities: the dashed line decribes the daily mean R-M effect and is the same as that shown at the base of Figure 1e.

for the effect would then be $\beta_{\mathrm{GSE}}$ and the relevant factor is $\mid \sin$ $\left(\beta_{\mathrm{GSE}}\right)$ ( (green lines): this variation peaks at the same time as the equinoctial variation. Various authors have used these phase differences for the predicted semi-annual variations to try to distinguish the mechanisms (e.g., Le Mouël et al., 2004) but, as pointed out by Russell \& McPherron (1973), a sufficiently large number of years must be used to remove statistical fluctuations caused by large transient events hitting Earth. Only then will the data reveal the true dates of the equinoctial peaks of the semiannual variation.

From studying the F-UT pattern for the am index, Cliver et al. (2000) inferred there was a mixture of all three mechanisms Nowada et al. (2009) concluded that a mixture of R-M and equinoctial effects was operating. Lockwood et al. (2016) have argued that axial-like elements are introduced by the integrating effect of the inherent response time of the storagerelease magnetospheric system and McPherron et al. (2013) and Chu et al. (2015) have inferred a combination of about $40 \%$ Russell-McPherron effect and 60\% equinoctial effect in the response of the auroral electrojet $A L$ index and a mid-latitude substorm index, respectively. 


\subsection{Power input to the magnetosphere}

In this series of papers we make extensive use of the power input to the magnetosphere, $P_{\alpha}$, which is computed from interplanetary measurements using the theoretical formulation by Vasyliunas et al. (1982). Lockwood et al. (2019a, b, c) argue that this is by far the most satisfactory of all solar wind magnetosphere coupling functions for studies of magnetospheric energetics, owing to its effectiveness while only having one free fit parameter, the coupling exponent $\alpha$ (hence it minimises the pitfall of "overfitting"). It is based on the dominant energy flux in the solar wind, the bulk flow kinetic energy of the particles, and neglects the Poynting flux, which makes up typically just $1 \%$ of the total solar wind energy flux (Lockwood, 2019). In comparison, the much-used epsilon factor is based on the incorrect idea that the energy density in the solar wind is in the form of Poynting flux and it performs less well on all averaging timescales $\tau$ as a result (Finch \& Lockwood, 2007). The formula for the Vasyliunas et al. (1982) power input into the magnetosphere is

$$
P_{\alpha}=\left(\pi c k_{2} k_{1}^{2} M_{\mathrm{E}}^{2 / 3} \mu_{0}^{-1 / 3}\right) m_{\mathrm{sw}}^{(2 / 3-\alpha)} N_{\mathrm{sw}}^{(2 / 3-\alpha)} V_{\mathrm{sw}}^{(7 / 3-2 \alpha)} B^{2 \alpha} \sin ^{4}(\theta / 2)
$$

where $m_{\mathrm{sw}}$ is the mean ion mass of the solar wind $N_{\mathrm{sw}}$ is the solar wind number density $V_{\mathrm{sw}}$ is the solar wind speed, $B$ is the IMF field strength and $\theta$ is the "clock angle" that the IMF vector makes with the $Z_{\mathrm{GSM}}$ axis in the $Z Y$ plane; $c$ is a dayside magnetospheric shape parameter, being the ratio of the cross sectional radius of the dayside magnetosphere $\left(l_{\mathrm{o}}\right)$ to the stand-off distance of the nose of the magnetosphere; $k_{1}$ is the magnetosheath pressure factor for supersonic flow around a blunt-nosed object; $k_{2}$ is a dimensionless constant in the solar-wind to magnetosphere transfer function; and $M_{\mathrm{E}}$ is the magnetic moment of the Earth which can be computed for a given time using the IGRF-15 Model (Thébault et al., 2015). Because the variation of $M_{\mathrm{E}}$ is with time is small and approximately linear we can treat the term in brackets as a constant that we can later cancel out by normalising $P_{\alpha}$ to its average value over the whole period $P_{\mathrm{o}}$ to give $P_{\alpha} / \mathrm{P}_{\mathrm{o}}$. The one free fit parameter is the coupling exponent $\alpha$.

By way of comparison, the epsilon factor (Perreault \& Akasofu, 1978) is

$$
\varepsilon=\left(l_{o}^{2} / 4 \pi\right) V_{\mathrm{sw}} B^{2} \sin ^{4}(\theta / 2) .
$$

So both $\varepsilon$ and $P_{\alpha}$ share the same IMF orientation factor, $\sin ^{4}(\theta / 2)$. The $\varepsilon$ parameter is often used because it does not require knowledge of the solar wind mean ion density nor number density: it avoids these terms by using a fixed constant value for the magnetosphere cross-sectional radius, $l_{\mathrm{o}}$, whereas $P_{\alpha}$ estimates this from pressure balance across the nose of the magnetosphere. As discussed above, the biggest difference is that $\varepsilon$ assumes that power arrives in the solar wind in the form of Poynting flux, whereas $P_{\alpha}$ is based on the kinetic energy density of the bulk flow of the solar wind. Typically, the kinetic energy density is $99 \%$ of the total solar wind energy compared to 1\% Poynting flux. However, Lockwood (2019) has used Poynting's theorem for a plasma to discuss how solar wind Poynting flux passes straight into the magnetosphere whereas kinetic energy flux has first to be converted into Poynting flux by currents flowing in the bow shock, magnetosheath and magnetopause. This means that solar wind Poynting flux contributes a larger fraction (about 10\%) of the total energy entering the magnetosphere. Consequently, Lockwood (2019) added solar wind Poynting flux to $P_{\alpha}$ predicted from the kinetic energy flux of the solar wind particles using equation (2) and showed that a small but significant improvement in the correlation with geomagnetic activity could be made for small averaging timescales $\tau$; however, the improvements were extremely small and were not statistically significant for $\tau$ greater than about 1 day. This also requires the use of a second empirical fit parameter which raises the danger of overfitting. Lockwood et al. (2019a) have shown that data gaps in the interplanetary data series introduce considerable noise into solar wind-magnetosphere coupling studies and so we here only use $P_{\alpha}$ for after 1995 when we have had almost continuous data on the near-Earth solar wind.

The formulations for the power input in both $P_{\alpha}$ and $\varepsilon$ use a factor $\sin ^{4}(\theta / 2)$ to account for the effect of IMF orientation on solar wind-magnetosphere coupling, where $\theta$ is the IMF "clock angle" in the GSM frame, i.e., $\theta=\arctan \left(\left|\left[B_{Y}\right]_{\mathrm{GSM}}\right| /\left[B_{Z}\right]_{\mathrm{GSM}}\right)$. Lockwood et al. (2019b) and Lockwood (2019) have shown that this $\sin ^{4}(\theta / 2)$ factor performs better than all suggested alternatives, including the "half-wave rectified" southward field which was the basis of the original R-M theory. The $\sin ^{4}(\theta / 2)$ factor preserves the non-linearity in the geomagnetic response, which is a vital factor in the R-M effect giving the semi-annual variation, but avoids the discontinuity in slope of $\left[B_{\mathrm{Z}}\right]_{\mathrm{GSM}}$ at $\theta=\pi / 2$ of the half-wave-rectified form. The factor also allows for a continued, lower rate of magnetopause reconnection opening geomagnetic field lines, even when the IMF is northward $(\theta<\pi / 2)$, as has been deduced in a number of studies, including observations of ionospheric $\mathrm{O}^{+}$ions escaping the magnetosphere on field lines opened by magnetic reconnection (Chandler et al., 1999). The relationship of the power input to the magnetosphere, based on the $\sin ^{4}(\theta / 2)$ factor, the halfwave-rectified $\left[B_{Z}\right]_{\mathrm{GSM}}$ of the R-M effect, and the IMF component perpendicular to the solar equatorial plane are discussed further in Appendix B and Section 3.

\subsection{Observed semiannual variations in power input into the magnetosphere and geomagnetic activity}

Figure 6 demonstrates the semi-annual variations in the geomagnetic indices and the estimated power input into the magnetosphere, $P_{\alpha}$, from equation (2). We use all available daily means of the $a a_{\mathrm{H}}$ index, $A a_{\mathrm{H}}$, which are for 1868-2017 (left), of the Dst index, available for 1957-2017 (middle) and of the power input into the manetosphere $P_{\alpha}$, which are reliable for 1995-2017 (right). The top panels show the mean values in 36 equal-width bins of $F$ (roughly 10 days width), with grey areas defining plus and minus one standard deviation. The lower panels show the mean number of days per year $N$ in which the daily means exceed the (blue) 90-percentile, (orange) 95-percentile, and (mauve) 99-percentile, respectively, of the overall distribution for the parameter in question.

The 3-hourly $a a_{\mathrm{H}}$ index and its daily means $A a_{\mathrm{H}}$ (like the corresponding am and Am indices) cannot detect individual substorm cycles which are only of order $1 \mathrm{~h}$ in duration. However, Appendix A uses the $A E$ and $A L$ auroral electrojet indices (Davis \& Sugiura, 1966), to demonstrate that the $A a_{\mathrm{H}}$ index is a good indicator of the level of substorm activity in each day (even if it cannot detect individual substorms), responding 


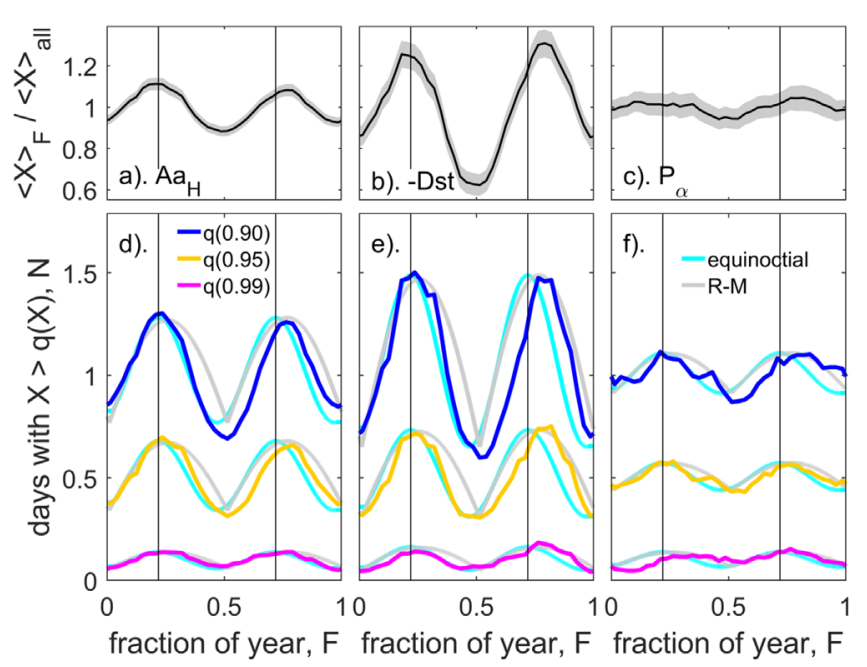

Figure 6. Annual variations in geomagnetic indices and power input into the magnetosphere: (left) the $A a_{\mathrm{H}}$ index means for 1868-2017; (middle) Dst index (1959-2017) and (right) daily the power input into the magnetosphere $P_{\alpha}$ (1995-2017). In the top panels, the black lines show the mean values in 36 equal-width bins of fraction of time-ofyear, $F$ (roughly 10 days width) and the grey areas are plus and minus one standard deviation: (a) $\left\langle A a_{\mathrm{H}}\right\rangle$; (b) $\langle D s t\rangle$; and (c) $\left\langle P_{\alpha}\right\rangle$. The lower panels show the variation of the number of days in which the daily means exceed the (blue) 90-percentile, (orange) 95-percentile, and (mauve) 99-percentile (d), (e) and (f) being for $A a_{\mathrm{H}}$, Dst and $P_{\alpha}$, respectively. The thin vertical lines mark the equinoxes (the times of the peaks in the equinoctial variation) and the grey and cyan are variations predicted by the Russell-McPherron and equinoctial effect, respectively, scaled in amplitude to match the observed variations so as to reveal waveform and phase differences.

primarily to the substorm current wedge. We also note that Dst is primarily, but not exclusively, an indicator or the magnetospheric ring current but that other currents, such as those in the magnetopause will have some effect.

The vertical black lines in Figure 6 mark the equinoxes (the peaks of the equinoctial effect, i.e. the vertical mauve lines in Fig. 5) and the cyan and grey lines are variations predicted in Figure 5 for the equinoctial and R-M effects, scaled in amplitude to match the observed variations so as to reveal phase differences (the axial and GSE effect variations are not shown to avoid over-complicating the plot). The upper panels of Figure 6 show the variations in mean values and the lower panels the variations in the occurrence of the index exceeding its 90,95 and 99 percentiles, respectively $q(0.9), q(0.95)$ and $q(0.99)$ : for $a a_{\mathrm{H}}$ these levels are $36.5 \mathrm{nT}, 47.0 \mathrm{nT}$ and $76.8 \mathrm{nT}$; for Dst they are $40.2 \mathrm{nT}, 53.8 \mathrm{nT}$ and $96.7 \mathrm{nT}$; and for $P_{\alpha} / P_{\mathrm{o}}$ they are 1.88, 2.30 and 3.62.

A few points stand out in Figure 6. In none of the geomagnetic datasets (covering different duration intervals) is there a consistent difference between the magnitudes of the two equinoctial peaks. However, there is a dominance of the September equinox in the $P_{\alpha}$ data which covers the shortest interval: hence to compare like-with-like we need to make comparisons over the same interval as the $P_{\alpha}$ data. Figures $1 \mathrm{~d}$ and 6 show that dominance of the September peak is not typical of the whole

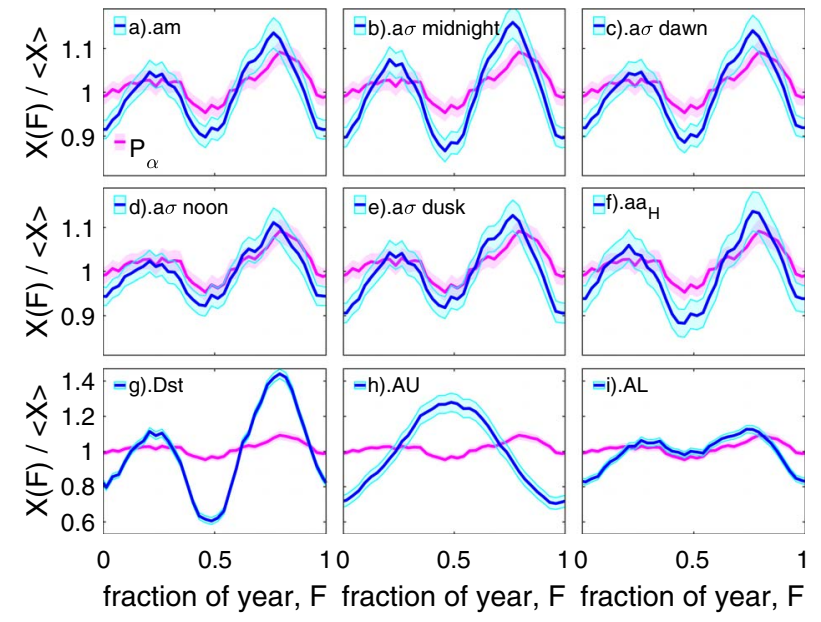

Figure 7. Comparison of the semi-annual variations in various geomagnetic indices (in blue) and the power input into the magnetosphere $\left(P_{\alpha}\right.$, in mauve). All data are all available 3-hourly values for the interval 1995-2017, inclusive and averages are taken in 36 bins of $F$ of width 0.0278 . The pink and cyan shaded errors are plus and minus one standard error in the means. In each case mean values of a generic index $X$ in each $F$ bin are normalized by dividing by the overall mean of $X$ over the whole interval. The panels are for the geomagnetic indices: (a) $a m$; (b) $a \sigma$-midnight; (c) $a \sigma$-dawn; (d) $a \sigma$-noon; (e) $a \sigma$-dusk; (f) $a a_{\mathrm{H}}$; (g) $D s t$; (h) $A U$ and (i) $A L$. Note that for the bottom row the $y$-scale has been expanded by a factor of 3 .

period of the $A a_{\mathrm{H}}$ data (1868-2017). Secondly, the axial effect matches neither the phase nor waveform of the observed variation, giving peaks that are consierably in advance from those observed. The peaks are closest to the times predicted by the $\mathrm{R}-\mathrm{M}$ effect. However, the waveform is much more like that predicted for the equinoctial effect. Thirdly, the semi-annual variation is present in $P_{\alpha}$, as expected for the R-M effect, but is of a much smaller amplitude than that for $A a_{\mathrm{H}}$, which is, in turn, smaller than that for Dst. This shows that either the magnetopshere has a second internal mechanism that causes a semiannual variation or it has a mechanism that amplifies the input variation in $P_{\alpha}$. In their original paper, Russell \& McPherron (1973) recognised that the fractional amplitude of the observed semi-annual variation in geomagnetic activity was considerably larger than that in the solar wind forcing, and although that amplification has been quantified in various subsequent papers, there has been no consensus on its origin (Cliver et al., 2000; de La Sayette, 2004; Weigel, 2007; Kuznetsova \& Laptukhov, 2011).

Figure 7 compares the amplitudes of the semi-annual variations in mean values (if seen) in a wide range of geomagnetic indices (shown in each panel in blue) with that for the power input into the magnetosphere $\left(P_{\alpha}\right.$, in mauve). All data are for the same interval, namely 1995-2017, (inclusive, i.e. data from the start and end years are both included). Each variation shows means in 36 equal-width bins of $F$, with an uncertainty band shaded in light colour that is plus and minus one standard error in the mean. In each case the fractional variation is shown by normalising to the overall mean value for the whole interval. In every case we see a semi-annual variation except in the 
eastward electrojet auroral index, $A U$, for which we see only an annual variation, peaking around the summer solstice (i.e. June and $F \approx 0.5$ ) as the auroral electrojet indices are measured in the northern hemisphere. Hence the variation with $F$ in $A U$ is dominated by ionospheric conductivity generated by photoionization. All other indices show a semi-annual variation and, for this interval, all show a dominant peak around the September equinox. From the 150-year data series of $a a_{\mathrm{H}}$ (Fig. 1), this appears to be a chance occurrence. The time of the peaks in all the geomagnetic indices are not significantly different from those in $P_{\alpha}$. The only exception to this is $A L$ which, although showing a semi-annual variation, also shows a strong annual variation with considerably lower values around the (northern hemisphere) winter solstice to those in summer. As for $A U$, this appears to be a conductivity effect in this northern hemisphere index and the annual variation it causes pushes both of the two peaks towards the summer solstice. The largest amplitude semiannual variation is in $D s t$, it being a larger than that in $P_{\alpha}$ by a factor of about 10. (Note that the $y$-axis scale in parts $\mathrm{g}$, $\mathrm{h}$, and $\mathrm{i}$ of Fig. 7 is three times larger than for the other six panels). This amplification is by a factor of two for the $a m, a \sigma$-dawn and $a \sigma$-dusk indices, but for $a \sigma$-noon it is only very slightly greater than unity, but is by a factor near three for $a \sigma$-midnight. This is consistent with the $F$-UT patterns presented for these indices by Chambodut et al. (2013) and implies strongly that the amplification of the semi-annual variation occurs on the nightside of the magnetosphere-ionosphere system. The large amplitude of the semi-annual variation in Dst has been noted previously (e.g., Mursula \& Karinen, 2005) and is discussed further in Section 6.

\section{Statistics of the am index as a function of Time-of-Year and UT}

Figure 8 plots the 288 cumulative distribution functions (c.d.f.s) of $a m /\langle a m\rangle_{\text {all }}$, where $\langle a m\rangle_{\text {all }}$ is the mean over all values in the dataset used, for 36 equal-sized bins of $F$ and the eight $U T$ intervals over which the index is derived. In (a) all available data are used (1959-2017), whereas in (b) data for the interval of near continuous interplanetary measurements are used (1995-2017) (see Lockwood et al., 2019a). The c.d.f.s are colour-coded according to the scale given by the values of $\langle a m\rangle_{\mathrm{UT,F}} /\langle a m\rangle_{\text {all }}$ where $\langle a m\rangle_{\mathrm{UT}, \mathrm{F}}$ is the mean value for the $F-U T$ bin in question. The black line is the c.d.f. for all data and the orange and green lines for the bin giving maximum and minimum $\langle a m\rangle_{\mathrm{UT}, \mathrm{F}}$, respectively. The distributions are all similar in shape and those in (a) are very similar to those in (b), although noise is a little greater in (b) than (a) because of the smaller number of samples. All results presented below for the full am interval were also generated for the shorter interval and the results are essentially the same and are not shown.

Figure 9 plots the quantiles of the distributions shown in Figure $8 \mathrm{a}$ as a function of $F$ and $U T$. We here use the notation that, for example, $q(0.5)$ is the 50-percentile (i.e., the median) of the variable in question and is exceeded $50 \%$ of the time (in that $F-U T$ bin). For all the quantiles shown, the equinoctial pattern is seen, except for the lowest displayed, $q(0.1)$.
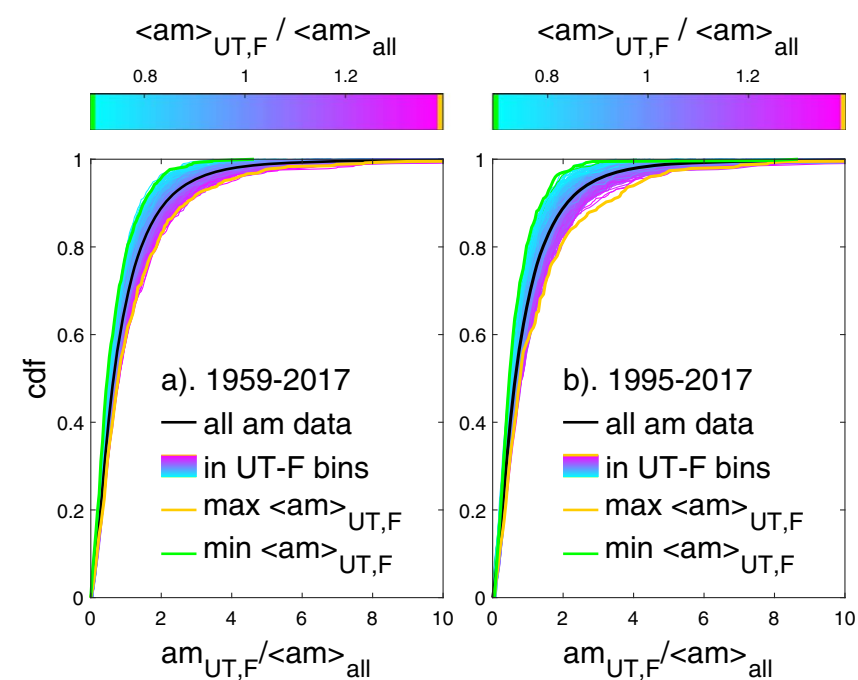

Figure 8. Cumulative probability distributions (c.d.f.s) of the $a m$ geomagnetic index, as a ratio of its mean value $\langle a m\rangle$, for data from (a) the full interval of am data (1959-2017) and (b) the interval of near-continuous interplanetary data (1995-2017). The am data are divided into 288 bins, 36 equal-sized bins in fraction of year $F$ and the 8 3-hour Universal Time $(U T)$ bins over which the range am index is evaluated. For each $F$-UT bin the c.d.f. of $a m$ as a ratio of the mean value for that bin $\left(\langle a m\rangle_{\mathrm{F}, \mathrm{UT}}\right)$ is plotted and colored according to the value of the ratio $\langle a m\rangle_{\mathrm{F}, \mathrm{UT}} /\langle a m\rangle_{\text {all }}$, where $\langle a m\rangle_{\text {all }}$ is the mean value for all 175,320 three-hourly am samples in the interval studied. The black line is for all am data, the orange line for the F-UT bin giving the largest $\langle a m\rangle_{\mathrm{F}, \mathrm{UT}}$ and the green line for the $F$-UT bin giving the smallest $\langle a m\rangle_{\mathrm{F}, \mathrm{UT}}$.

However, note that the 0.99 percentile has only 1752 samples which is an average of just six samples in each bin and it is rather remarkable that even at these low sample numbers an equinoctial $F-U T$ pattern can still be seen. For all quantiles up to approximately $q(0.8)$, the March equinox dominates over the September 1 at 9-24 UT, but September dominates for 0-6 UT, for $q(0.8)$ the two peaks are roughly equal at all UT and for the very largest values, $q(0.99)$, the September dominates at 12-21 UT whereas the March dominates at 21-12 UT, almost the opposite behaviour seen in lower quantiles. This behaviour is hidden in the average am values and the full distribution (Fig. 9a), for which the two equinox peaks are roughly equal in magnitude at all UT. Therefore, for the period shown (since 1959), the March equinox has dominated at moderate-to-large activity levels, but there have been some extremely large events at the September equinox (mainly at 12-21 UT) that have influenced the relative sizes of the two equinox peaks. We have no way of knowing if this behaviour is typical in the longer term because $a m$ is the only index that has a sufficiently uniform $F-U T$ response (Lockwood et al., 2019d) and is only available for the interval used to compile Figure 9 (1959-2018).

In Figure 9, the means show lower values at 03-09 UT at both equinoxes and this is also seen for all quantiles. This will be discussed further in Section 5. 


\section{$\mathrm{am} /<\mathrm{am}>1959-2017$}
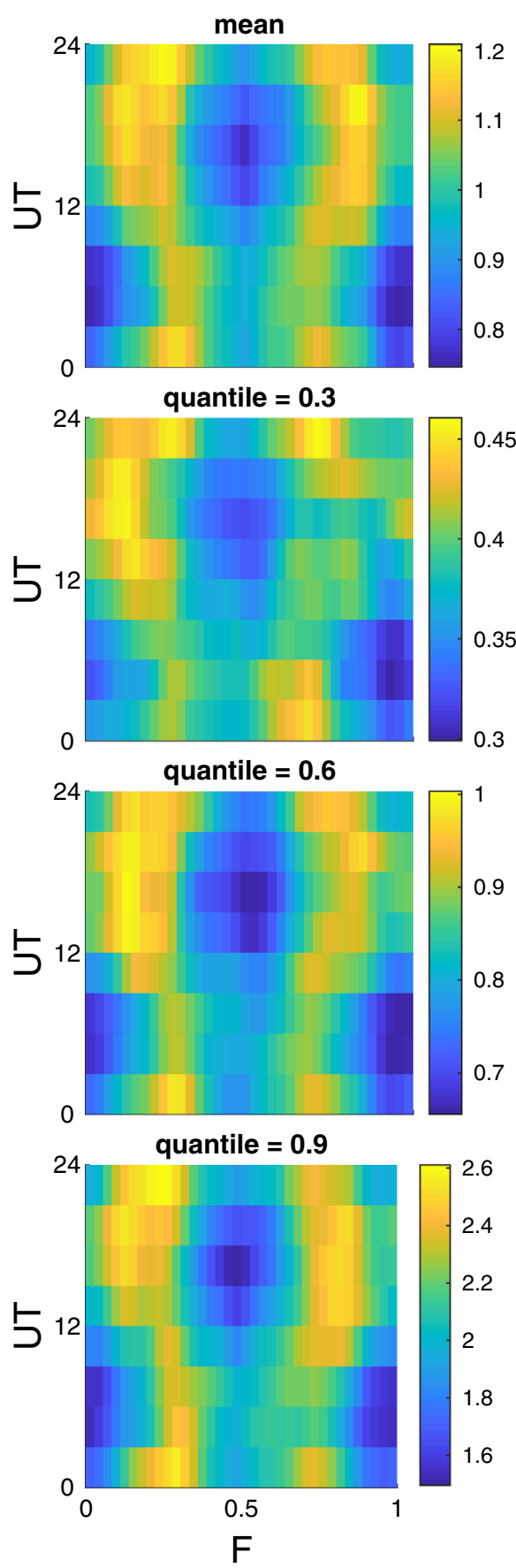

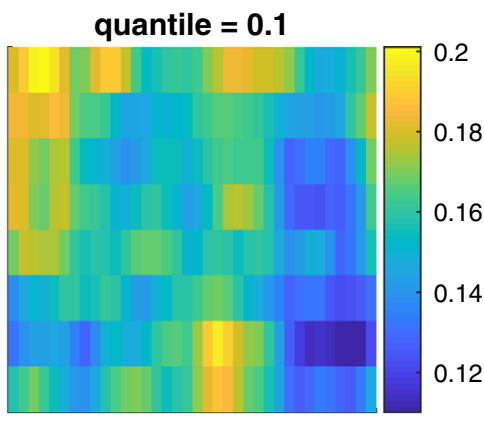

quantile $=0.4$

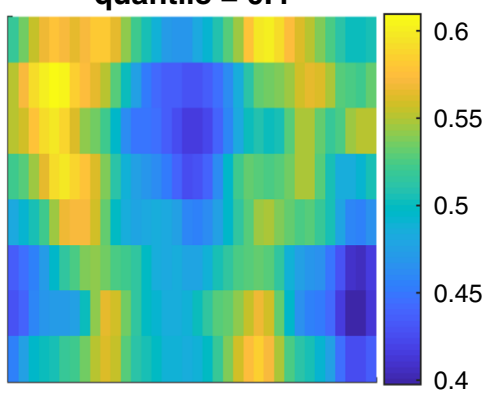

quantile $=0.7$

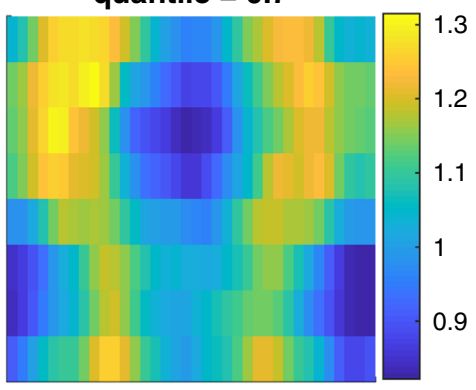

quantile $=0.95$

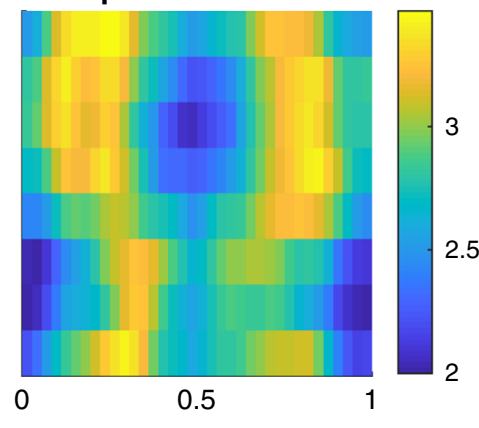

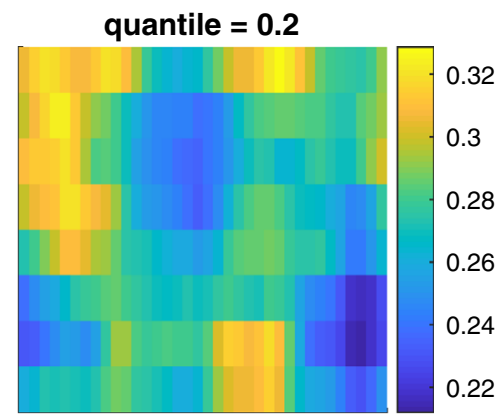

quantile $=0.5$

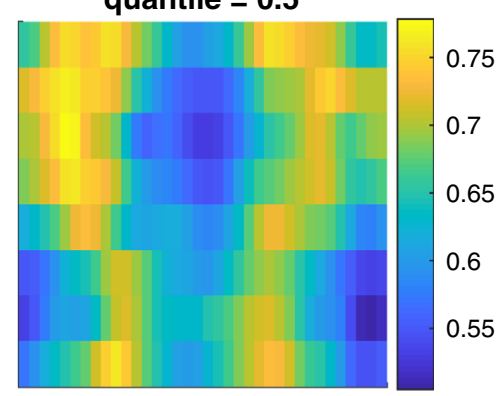

quantile $=0.8$

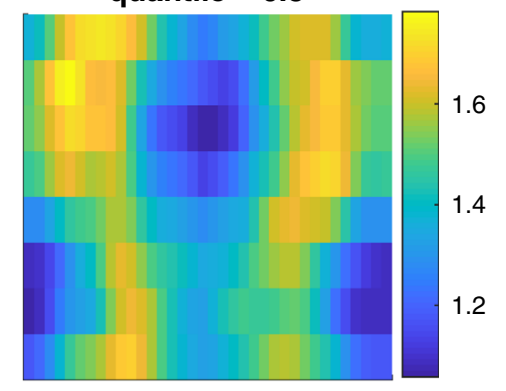

quantile $=0.99$

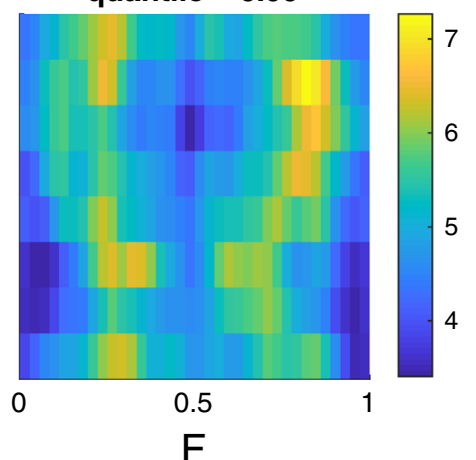

Figure 9. Time-of-year/time-of-day $(F-U T)$ plots of: (a) the am index, $\langle a m\rangle_{\mathrm{F}, \mathrm{UT}}$ averaged in the 288 F-UT bins used in Figure 8; (b)-(1) the quantiles of the $a m /\langle a m\rangle_{\text {all }}$ distribution in each F-UT bin: (b) $q(0.1)$; (c) $q(0.2)$; (d) $q(0.3)$; (e) $q(0.4)$; (f) $q(0.5)$; (g) $q(0.6)$; (h) $q(0.7)$; (i) $q(0.8$ ); (j) $q(0.9)$; (k) $q(0.95)$; and (1) $q(0.99)$. All finalised am data are used which are from 1959 to 2017, inclusive.

\section{The relative contributions of the Russell- McPherron effect and out-of-ecliptic field}

In this section, we investigate the relative contributions of southward field in the GSEQ frame (also giving strong outof-ecliptic field in the GSE frame) and of the R-M effect to power input into the magnetosphere and to the semi-annual variation. To do this we look at the "half-wave-rectified" southward field in a general rest frame RF, $\left[B_{\mathrm{S}}\right]_{\mathrm{RF}}$ defined by:

$$
\begin{aligned}
& {\left[B_{\mathrm{S}}\right]_{\mathrm{RF}}=-\left[B_{Z}\right]_{\mathrm{RF}} \text { for }\left[B_{Z}\right]_{\mathrm{RF}}<0} \\
& {\left[B_{\mathrm{S}}\right]_{\mathrm{RF}}=0 \text { for }\left[B_{Z}\right]_{\mathrm{RF}} \geq 0}
\end{aligned}
$$



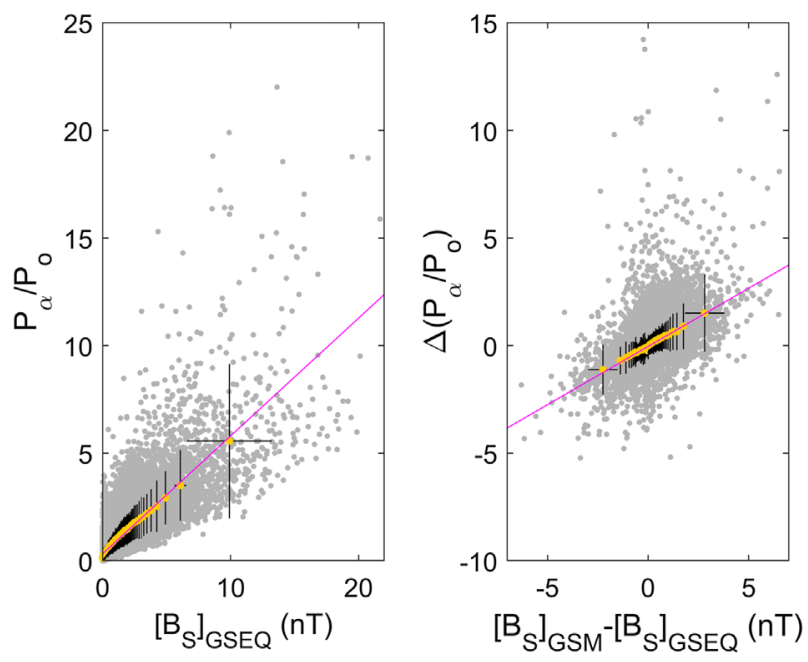

Figure 10. (a) Scatter plot of normalized power input to the magnetosphere, $P_{\alpha} / P_{\mathrm{o}}$, as a function of southward field in the GSEQ frame of reference, $\left[B_{\mathrm{S}}\right]_{\mathrm{GSEQ}}$, defined by $\left[B_{\mathrm{S}}\right]_{\mathrm{GSEQ}}=-\left[B_{Z}\right]_{\mathrm{GSEQ}}$ for $\left[B_{Z}\right]_{\mathrm{GSEQ}}<0$ and $\left[B_{\mathrm{S}}\right]_{\mathrm{GSEQ}}=0$ for $\left[B_{Z}\right]_{\mathrm{GSEQ}}>0$. Grey dots are for 3 -hourly means, orange dots are means in 1-percentile ranges of $\left[B_{\mathrm{S}}\right]_{\mathrm{GSEQ}}$ and error bars are plus and minus one standard deviation in those ranges. The mauve line is a linear regression fit to the 3-hourly means. (b) The same as (a) for the fit residuals in (a), $\Delta\left(P_{\alpha} / P_{\mathrm{o}}\right)$, as a function of the difference in the half-wave-rectified southward fields in the GSEQ and GSM frames, $\left[B_{\mathrm{S}}\right]_{\mathrm{GSEQ}}-\left[B_{Z}\right]_{\mathrm{GSM}}$. Data are from 1995 to 2017 , inclusive.

Appendix B compares the $\left[B_{\mathrm{S}}\right]_{\mathrm{GSM}} / B$ and $\sin ^{4}(\theta / 2)$ IMF orientation factors and how they vary with the dipole tilt to give the R-M effect: the former was used in the original paper by Russell $\&$ McPherron (1973) the latter is used by the $P_{\alpha}$ and $e$ coupling functions. To evaluate the importance of using the GSM frame, we compare how $\left[B_{\mathrm{S}}\right]_{\mathrm{GSM}}$ and $\left[B_{\mathrm{S}}\right]_{\mathrm{GSEQ}}$, the half-wave-rectified southward field in the GSM and GSEQ frames computed using equation (4), relate to the normalised power input into the magnetosphere $P_{\alpha} / P_{\mathrm{o}}$. We use 3-hourly averages of IMF data to compare with the 3-hourly am values but shift the averaging intervals forward by $1 \mathrm{~h}$ to allow for the average response lag of am (this response lag will be discussed in detail in a later paper). The grey points in Figure 10a give a scatter plot of $P_{\alpha} / P_{\mathrm{o}}$ as a function of $\left[B_{\mathrm{S}}\right]_{\mathrm{GSEQ}}$. It can be seen that there is considerable scatter, but also a good linear correlation between the two. The mauve line is a linear regression fit to these data. The orange points and corresponding black error bars show, respectively, the means and standard deviations of $P_{\alpha} / P_{\mathrm{o}}$ in the 99 one-percentile-wide bins of $\left[\mathrm{B}_{\mathrm{S}}\right]_{\mathrm{GSEQ}}$.

This demonstrates that large southward field in the GSEQ frame is the major driver of events of large power input into the magnetosphere. We expect the R-M effect to be a major contributor to the scatter in these data, in addition to other contributors that include variations in solar wind speed, IMF magnitude, number density and mean ion mass. To quantify the scatter we compute the fit residuals between each $P_{\alpha} / P_{\mathrm{o}}$ data point and the best fit linearly regressed value (the mauve line in Fig. 10a) at the same $\left[B_{\mathrm{S}}\right]_{\mathrm{GSEO}}, \Delta\left(P_{\alpha} / P_{\mathrm{o}}\right)$. These are plotted in Figure $10 \mathrm{~b}$ as a function of $\left(\left[B_{\mathrm{S}}\right]_{\mathrm{GSM}}-\left[B_{\mathrm{S}}\right]_{\mathrm{GSEQ}}\right)$, which is
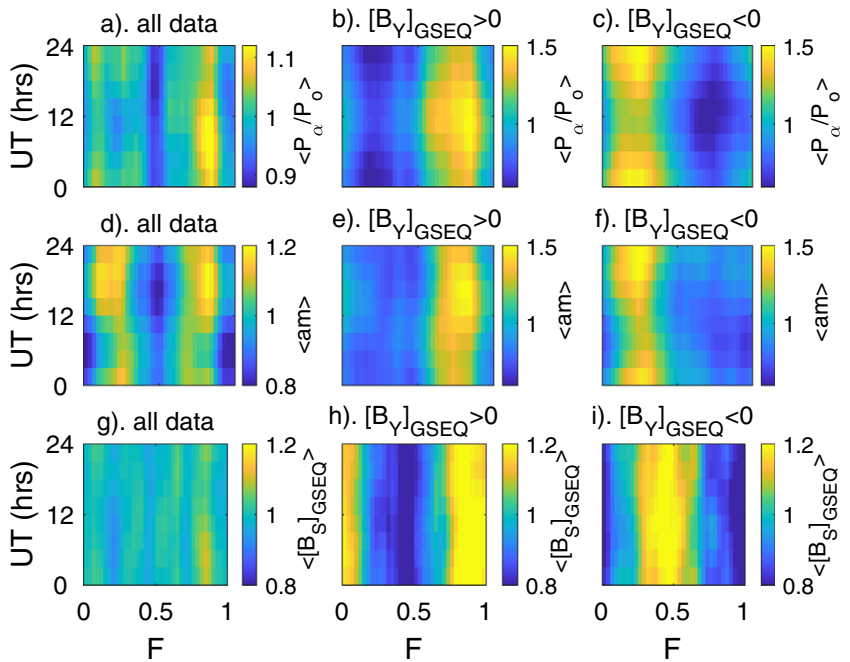

h). $\left[B_{Y}\right]_{G S E Q}>0$
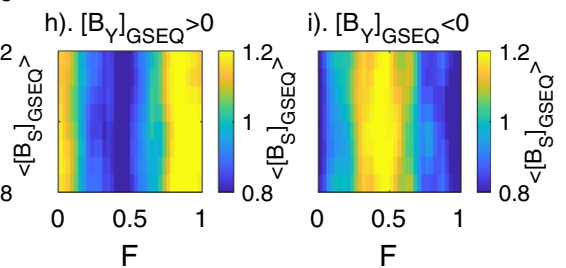

Figure 11. F-UT patterns of mean values for (top row) the normalized power input into the magnetosphere, $P_{\alpha} / P_{\mathrm{o}}$; (middle row) the am index and (bottom row) the southward field in the GSEQ frame, $\left[B_{\mathrm{S}}\right]_{\mathrm{GSEQ}}$. The data are 3-hour averages for 1995-2017, inclusive, and are averaged into 36 equal sized bins in $F$ and the 8 UT time ranges over which the $a m$ index is derived. For the IMF data used $\left(P_{\alpha} / P_{\mathrm{o}},\left[B_{Y}\right]_{\mathrm{GSEQ}},\left[B_{\mathrm{S}}\right]_{\mathrm{GSEQ}}\right)$ the 3 -hour averaging windows were moved forward by $1 \mathrm{~h}$ to allow for the average response delay of am to the interplanetary conditions. Plots in the left-hand column are for all data, plots in the middle column are for $\left[B_{Y}\right]_{\mathrm{GSEQ}}>0$, and plots in the right-hand column are for $\left[B_{Y}\right]_{\mathrm{GSEQ}}<0$.

the contribution of the R-M effect to the southward IMF component in the GSM frame. It can be seen that the R-M effect can both reduce and increase the southward component (as expected, depending on the polarity of $\left[B_{Y}\right]_{\mathrm{GSEQ}}$ and $F$ ) and there is again an approximately linear relationship. However, we can see an asymmetry as the enhancements tend to be greater than the decreases. The lowest $1 \%$ of $\left[B_{\mathrm{S}}\right]_{\mathrm{GSEQ}}$ samples in Figure 10a, i.e. the quantile range between $q(0)$ and $q(0.01)$, gives a mean value of $P_{\alpha} / P_{\mathrm{o}}$ of 0.12 whereas the largest $1 \%$, between $q(0.99)$ and $q(1)$, gives a mean of 5.57 and hence the range of variation in $\left[B_{\mathrm{S}}\right]_{\mathrm{GSEQ}}$, attributable to transients giving out-of-equatorial heliospheric field, causes a variation in $P_{\alpha} / P_{\mathrm{o}}$ of order 5.45. In contrast, the lowest $1 \%$ of $\left(\left[B_{\mathrm{S}}\right]_{\mathrm{GSM}}-\left[B_{\mathrm{S}}\right]_{\mathrm{GSEQ}}\right)$ samples in Figure $10 \mathrm{~b}$ gives a mean value of the fit residual $\Delta\left(P_{\alpha} / P_{\mathrm{o}}\right)$ of -1.11 whereas the largest $1 \%$ gives a mean of +1.51 and hence the range of variation in $\left(\left[B_{\mathrm{S}}\right]_{\mathrm{GSM}}-\left[B_{\mathrm{S}}\right]_{\mathrm{GSEQ}}\right)$ attributable to the R-M effect causes a variation in $P_{\alpha} / P_{\mathrm{o}}$ of order 2.62. Hence we find that the average contribution of the R-M effect to power input into the magnetosphere, and hence geomagnetic activity, is about half of that due to southward field in the GSEQ frame.

Figure 10 shows that both southward field in the GSEQ frame and the R-M effect contribute to enhanced power input into the magnetosphere. However, this does not tell us about the relative contributions of these two factors to the semi-annual variation in geomagnetic activity. This is analysed in Figures 11 and 12 for the near-continuous interplanetary data for 1995 2017, inclusive. To do this we search for the unique feature of the R-M effect that $\left[B_{Y}\right]_{\mathrm{GSEQ}}>0$ gives enhanced power input to the magnetosphere (and hence geomagnetic activity) around 

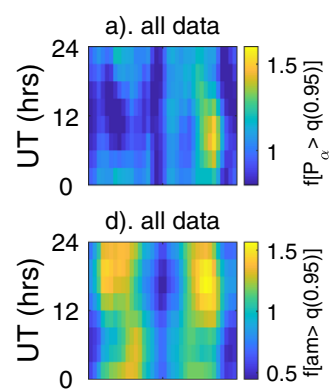

b). $\left[\mathrm{B}_{\mathrm{Y}}\right]_{\mathrm{GSEQ}}>0$

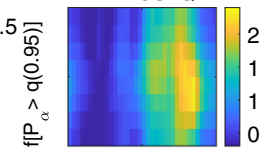

e). $\left[B_{Y}\right]_{G S E Q}>0$ c). $\left[\mathrm{B}_{\mathrm{Y}}\right]_{\mathrm{GSEQ}}<0$

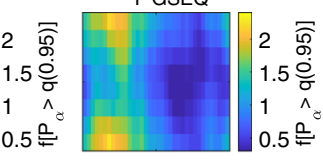

f). $\left[B_{Y}\right]_{G S E Q}<0$
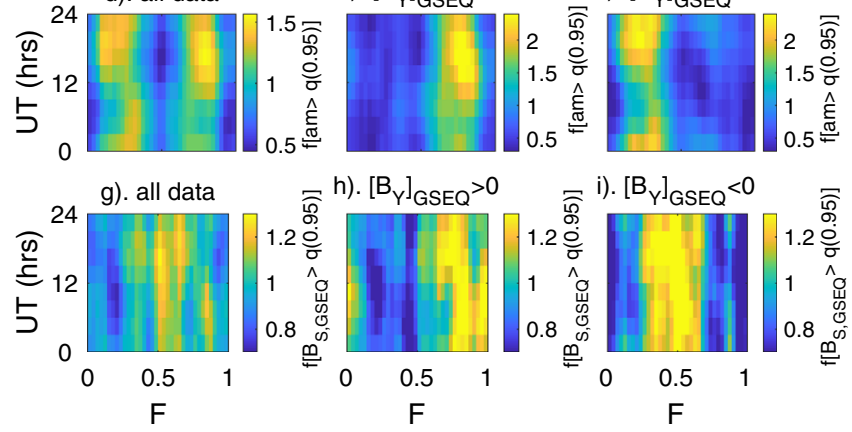

Figure 12. The same as Figure 11, for occurrence frequencies of large events, defined as exceeding the 95-perecentile of that parameter. Hence the top row shows the occurrence frequency of the 3-hourly means of the power input to the magnetosphere exceeding their 95-percentile, $f\left[P_{\alpha}>q(0.95)\right]$, the middle row shows the corresponding occurrence frequency for the am index, $f[a m>q$ (0.95)]; and the bottom row shows the corresponding occurrence frequency for $\left[B_{\mathrm{S}}\right]_{\mathrm{GSEQ}}, f\left[B_{\mathrm{S}, \mathrm{GSEQ}}>q(0.95)\right]$.

the September equinox, whereas $\left[B_{Y}\right]_{\mathrm{GSEQ}}<0$ gives the same around the March equinox. As discussed in the introduction, this also removes the effect of the Rosenberg-Coleman (R-C) mechanism which only changes the relative numbers of samples in the two $\left[B_{Y}\right]_{\text {GSEQ }}$ polarity subsets and at and around sunspot minimum. Figure 11 analyses the $F-U T$ patterns in mean values, whereas Figure 12 studies large events by looking at the occurrence of the parameters in the top $5 \%$ of values (for each $F-U T$ bin): in both cases data subsets for positive and negative $\left[B_{Y}\right]_{\mathrm{GSEQ}}$ are considered. The top rows are for the normalised power input, $P_{\alpha} / P_{\mathrm{o}}$ : for the all-data set, the R-M pattern is present but is not at all clear, especially in the mean values (it is somewhat clearer for the occurrence of values exceeding the 95th percentile $f\left[P_{\alpha}>q(0.95)\right]$ in Fig. 12a). However, splitting the data into $\left[B_{Y}\right]_{\mathrm{GSEQ}}>0$ and $\left[B_{Y}\right]_{\mathrm{GSEQ}}<0$ subsets reveals the two halves of the pattern very clearly indeed with peaks in power input at the expected $U T$ for each equinox. The middle rows of these figures shows the am responses. Figures $11 \mathrm{~d}$ and $12 \mathrm{~d}$ reveal the equinoctial pattern and $11 \mathrm{e}, 11 \mathrm{f}, 12 \mathrm{e}, 12 \mathrm{f}$ reveal the two halves of the equinoctial pattern separately. These plots show conclusively that almost all of the semi-annual variation in am is driven by the R-M effect. The bottom rows show the variations for $\left[B_{\mathrm{S}}\right]_{\mathrm{GSEQ}}$. For all data there is very little coherent structure, but for $\left[B_{Y}\right]_{\mathrm{GSEQ}}>0$ and $\left[B_{Y}\right]_{\mathrm{GSEQ}}<0$ an axial structure emerges. Because Parker spiral configuration applies on average, these broadly correspond to $\left[B_{X}\right]_{\mathrm{GSEQ}}<0$ and $\left[B_{X}\right]_{\text {GSEQ }}>0$, respectively. This gives an axial effect in the mean values shown in Figures $10 \mathrm{~h}$ and $10 \mathrm{i}$ which is consistent with the R-C effect and the fact that the two solar cycles covered in the interval are of very different amplitudes, giving asymmetries in the $\left[B_{X}\right]_{\mathrm{GSEQ}}$ sampled. The increased number of geoeffective transient events in September for $\left[B_{Y}\right]_{\mathrm{GSEQ}}>0$ (Fig. 12h) appears to be a random chance occurrence which explains the
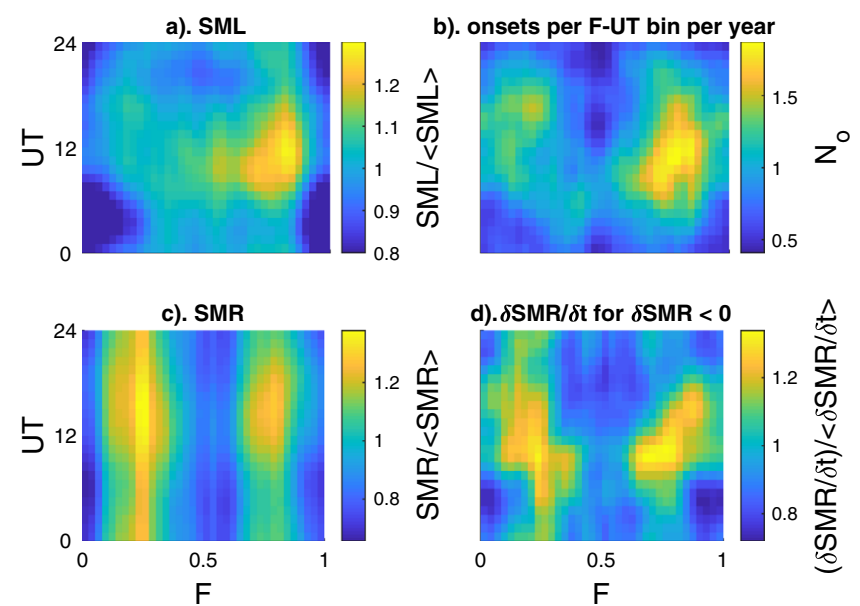

Figure 13. Time-of-year/time-of-day $(F-U T)$ plots of (a) the $S M L$ index; (b) the number of substorm onsets $N_{\mathrm{o}}$, per $F$-UT bin per year defined using $S M L$ and $P_{\alpha}$; (c) the $S M R$ index and (d) the average rate of decreases in of SMR. Data are for 1995-2017, inclusive, and in cases, values in a given $F$-UT bin are normalized to the overall mean value.

dominance of the September peaks at these times (but as shown by the long $a a_{\mathrm{H}}$ data series in Fig. 1, not at all times).

These results for each IMF $\left[B_{Y}\right]_{\mathrm{GSEQ}}$ polarity separately confirm that the Russell-McPherron effect is at the core of the semiannual variation in geomagnetic activity. However, Figure 10 shows that southward field normal to the solar equatorial plane is a larger factor (by a factor of about 2) in driving geomagnetic activity. We expect this because there is a universal understanding that CMEs and CIRs deflecting the field in this direction are major drivers of space weather events. The semi-annual variation in magnetospheric power input is relatively weak and the semi-annual variation is amplified in geomagnetic activity, a fact that was recognised by Russell \& McPherron (1973) in their original paper. In later papers in this series we will study this amplification.

\section{F-UT patterns in other Geomagnetic Indices}

As discussed in Section 1, the flat nature of the F-UT response pattern for the am index makes it the best index for defining the true F-UT variation in geomagnetic activity. However, information is still available from other indices if we consider the limitations that the distribution of stations places on them. Figure 13a shows the F-UT pattern for all available $S M L$ index data for all available data between 1974 and 2017. The $S M L$ index is generated by the SuperMAG project and is equivalent to $A L$ of the Auroral Electrojet indices (Newell \& Gjerloev, 2011a, b) but, whereas $A L$ is the lowest value seen by a ring of 12 northern hemisphere auroral stations, SML is the lowest value of the background-subtracted northward component seen in the global network of (typically) 110 northern hemisphere stations, between $+40^{\circ}$ and $+80^{\circ}$ degrees geomagnetic latitude. Because it is made from northern hemisphere stations only, as for $A L$, the equinoctial $F-U T$ pattern is convolved with a seasonal variation which inflates values in 
Northern hemisphere summer $(F=0.5)$ when ionospheric conductivities are enhanced by photoionization (as seen in Figure $8 \mathrm{i}$ for the $A L$ index). The variation in Figure $13 \mathrm{a}$ is similar to that found in Plate 4 of Cliver et al. (2000) for the $A E$ index, which is also a northern hemisphere index and so also has a spurious enhanced values around $F=0.5$.

A product that can be generated from the $S M L$ index is the time of substorm onsets, $t_{\mathrm{o}}$. These are defined by the SuperMAG project using the algorithm that the $S M L$ value must drop by $15 \mathrm{nT}$ in three successive minutes and subsequently remain at least $100 \mathrm{nT}$ below the value at $t_{\mathrm{o}}\left(S M L_{\mathrm{o}}\right)$ for the subsequent $25 \mathrm{~min}$. In other words $S M L<S M L_{\mathrm{o}}-15 \mathrm{nT}$ at $\left(t_{\mathrm{o}}+1 \mathrm{~min}\right)$, $S M L<S M L_{\mathrm{o}}-30 \mathrm{nT}$ at $\left(t_{\mathrm{o}}+2 \mathrm{~min}\right), \mathrm{SML}<S M L_{\mathrm{o}}-45 \mathrm{nT}$ at $\left(t_{\mathrm{o}}+3 \mathrm{~min}\right)$ and $S M L<S M L_{\mathrm{o}}-100 \mathrm{nT}$ over the interval between $\left(t_{\mathrm{o}}+4 \mathrm{~min}\right)$ and $\left(t_{\mathrm{o}}+29 \mathrm{~min}\right)$. To ensure that the substorms follow a well-defined growth phase, we here also require that the mean value of the power input to the magnetosphere $P_{\alpha}$, derived from interplanetary data as described above, be more than twice its overall average value $\left(P_{\mathrm{o}}\right)$ during the $20 \mathrm{~min}$ before $t_{\mathrm{o}}$ i.e. $P_{\alpha} / P_{\mathrm{o}} \geq 2$ for between $\left(t_{\mathrm{o}}-21 \mathrm{~min}\right)$ and $\left(t_{\mathrm{o}}-1 \mathrm{~min}\right)$. The resulting time series of the number of onsets per year follows very closely the annual mean sunspot number and the annual means of indices such as the $S M L, A E$ and $a m$ (Tanskanen, 2009; Chu et al., 2015). Figure 13b shows the number of onsets per year, per F-UT bin: it is not an ideal equinoctial $F-U T$ variation, but it has some clear similarities.

Figure $13 \mathrm{c}$ shows the variations of the SuperMAG SMR index (Newell \& Gjerloev, 2012), this is a ring current index that is based on the SYM-H index. It employs backgroundsubtracted northward component measured at a network of typically 100 stations at geomagnetic latitudes $\Lambda_{M}$ between $-50^{\circ}$ and $+50^{\circ}$. A $1 / \cos \left(\Lambda_{\mathrm{M}}\right)$ normalisation is then applied to give values close to what would have been observed by a station at the magnetic equator and the same Magnetic Local Time (MLT). All available data in four 6-hour MLT sectors (centered at $00,06,12$, and $18 \mathrm{~h} \mathrm{MLT)}$ are averaged together and these four means are then averaged to give $S M R$. Figure $13 \mathrm{c}$ shows that the $F-U T$ pattern for $S M R$ is primarily axial, as is that for the Dst index, once allowance is made for the uneven longitudinal spacing of the Dst stations (see Fig. $2 \mathrm{f}$ of Lockwood et al., 2016). As discussed above, Lockwood et al. (2016) show this is to be expected for the long integration time of solar wind forcing to which large storms in Dst are a response. Cliver et al. (2000) found that the time differential of Dst had indications of an equinoctial $F-U T$ pattern, (their Plate 3 ) and Figure $12 \mathrm{~d}$ shows the mean rate of decreases in $S M R(\mathrm{~d} S M R / \mathrm{d} t$ for $\mathrm{d} S M R<0$ ) shows a marked equinoctial pattern. The decreases in $S M R$ are times when injection into the ring current is dominating over the loss processes. Hence the limitations and characteristics of geomagnetic indices other than am mean that they do not all show an equinoctial pattern but with analysis which allows for these, the equinoctial pattern can often be identified.

\section{UT variation in the magnetosphere}

Many past papers have noted a UT variation in geomagnetic activity, particularly in the am index (Russell, 1989; de La Sayette \& Berthelier, 1996; Cliver et al., 2000) and the Auroral Electrojet indices $A E$ and $A L$ (Davis \& Sugiura, 1966; Allen \&
Kroehl, 1975; Hajkowicz, 1992, 1998; Ahn et al., 2000; Ahn \& Moon, 2003) with lower activity at 3-9 UT. There has been a concern with all these studies that the UT variation may have arisen out of spatial inhomogenities in the network of stations and seasonal photon-generated conductivity variations (particularly for the $A E$ indices, for which the stations are all in the northern hemisphere); although both limitations were judged inadequate explanations of the observed $U T$ variations by the above authors. Even the $F$-UT plots for the $a a_{\mathrm{H}}$ index presented in Figure 13 of Lockwood et al. (2018b) show the 03-09 UT minimum (both for the 1959-2017 plot, which can be compared with the am data, and for the prior 1868-1958 data). This finding must be put in context: $a a_{\mathrm{H}}$ is based on just two stations and correcting for this limitation to its $U T$ response depends upon the model of the station sensitivities. However, this model has successfully retrieved the equinoctial pattern $F-U T$ pattern for both intervals (seen for the 1959-2017 data in am but not seen in the classic $a a$ data) which implies the model correction is of value. With this caveat about the accuracy of the model station sensitivity correction, the $a a_{\mathrm{H}}$ data provide evidence that the 03-09 UT minimum has been present since 1868. The implications of this being a real effect in magnetosphere could be considerable for space-weather predictions - for example Hajkowicz (1992) finds that it drives considerable $U T$ variation in the occurrence of major ionospheric storms. Given that larger disturbances happen in the midnight MLT sector, this means geomagnetic activity may be systematically greater at some longitudes. Variations with $U T$ (and hence longitude) are well known in ionosphere-thermosphere coupling because the neutral thermosphere is oriented with respect to Earth's rotational poles whereas the ionospheric plasma is ordered relative to the geomagnetic poles, and several $U T$ variations result from the offset between the two (e.g. Klimenko et al., 2007). Another known source of $U T$ variation is associated with particle precipitation and longitudinal structure in Earth's magnetic field: the most striking example being trapped energetic particle precipitation at preferred longitudes because of the South Atlantic Anomaly (e.g., Pinto \& Gonzalez, 1989).

The papers discussed above and a number of the figures in the present paper point to a minimum in geomagnetic activity in the interval 00-09 UT. This minimum can be seen for all $F$ in Figure 9 in all the am quantiles that show the equinoctial effect (i.e., in all but $q(0.1)$, the lowest quantile shown). It is also present the mean am values. In Figure 13 it is also seen in plots derived from the $S M L$ and $S M R$ indices and is even true for the otherwise axial-like $F-U T$ pattern for SMR. The large numbers of stations used in generating both $S M L$ and $S M R$ indices helps reduce the concerns about the effect of nonuniformity in the distribution of stations but does not remove them. In this section we use the modelling of the response of the am index (and its components an and as) by Lockwood et al. (2019d) with the observations, to investigate the reality of the $U T$ variation.

The left hand column of Figure 14 studies average conditions, right-hand column studies the occurrence of large events. In each panel, all the values are normalised by dividing by the average over all $U T$ so that the relative amplitudes of the $U T$ variations can be compared. We make use of the concept of the sensitivity of individual stations to solar forcing, and hence of a network of stations used to compile an index as used by Lockwood et al. (2019d). The location-dependent 


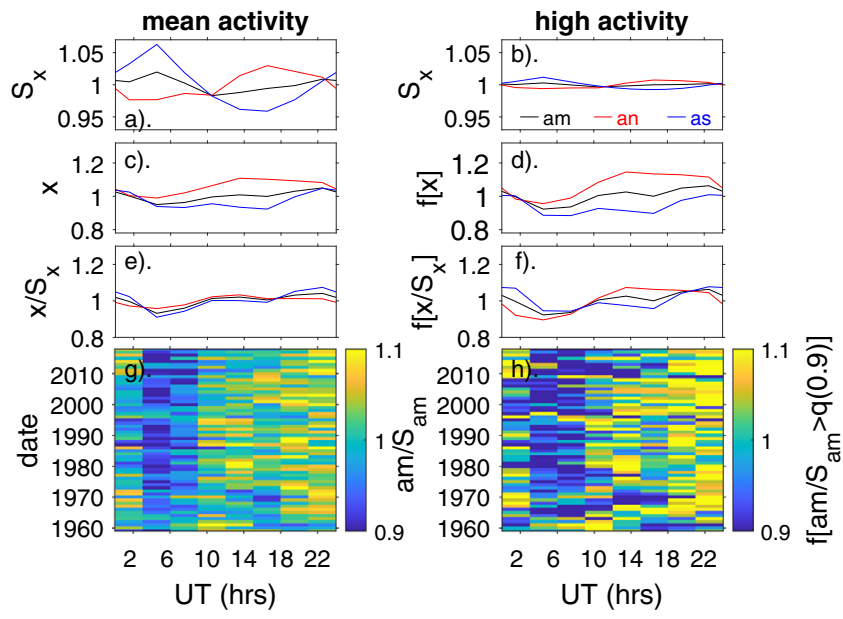

Figure 14. Universal Time (UT) variations of the am index and of its northern and southern hemisphere components, an and as. The lefthand plots are for averages and the right-hand plots are for the frequency of high activity events, defined by am exceeding its 90-percentile, $q(0.9)=44 \mathrm{nT}$. Black lines are for $a m$, red for $a n$ and blue for as. Parts (a) and (b) show the sensitivities of the three indices, $S_{x}$, computed using the model of Lockwood et al. (2019d) and averaged over all $F$ for each of the 8 UTs: (a) is for $a m=\langle a m\rangle=21 \mathrm{nT}$ and (b) is for $a m=q(0.9)=44 \mathrm{nT}$. (c) The indices averaged over all years and all $F$ for the 8 UT bins (am, an and as are given the generic axis label $x$ ); (d) corresponding occurrence frequency $f$ of the index $x$ exceeding its 90-percentile, $f[x>q(0.90)]$; (e) The indices allowing for modelled sensitivity $x / S_{x}$. (f) The large event occurrence frequencies allowing for index sensitivity, $f\left[x / S_{x}>q(0.90)\right]$. (g) and (h) The mean $\mathrm{am} / \mathrm{S}_{\mathrm{am}}$ and $f[\mathrm{am} /$ $\left.S_{\mathrm{am}}>q(0.90)\right]$, respectively, for each year separately, as a function of UT and year. Data are for 1959-2017, inclusive.

magnetometer station sensitivity, $s$, will also depend on $F$ and $U T$ and is defined simply for any given type of single-station geomagnetic activity measure $g$ by

$$
s=\frac{g}{I_{\mathrm{S}}}
$$

where $I_{\mathrm{S}}$ some measure of the input solar forcing (which includes the effects of both induced currents in near-Earth space driven by solar wind-magnetosphere coupling and of conductivity changes due to the flux of ionizing EUV and $\mathrm{X}$-ray radiations from the Sun or due to particle precipitation): $s$ is defined to be a function of only the instrument co-ordinates because instrument and local site characteristics are accounted for by other inter-calibration procedures. By taking ratios of $g$ seen simultaneously at many pairs of different stations, the $I_{\mathrm{S}}$ factor is cancelled and the ratios of the station sensitivities is obtained. Because the station responses are generally non-linear functions of $I_{\mathrm{S}}$, we find that we also need to allow for the activity level: we achieve this using the value of the combined geomagnetic index $G$. Note that this concept is the same as was used by Bartels (1949) and is still used today in the compilation of the $a p$ and $k p$ indices via look-up tables that give a ratio of station sensitivities for a given location as a function of $F, U T$ and the value of $g$ measured at that station. If the data from different stations are combined into a geomagnetic index using linear mathematics, then the sensitivities are similarly combined. For example, if the $g$ data from $N$ stations are averaged together with weighting functions $\omega$ to give the planetary index $G$,

$$
G=\sum_{i=1}^{N} \frac{\omega_{i} g_{i}}{N}=\sum_{i=1}^{N} \frac{\omega_{i} s_{i} I_{\mathrm{S}}}{N}=I_{\mathrm{S}} \sum_{i=1}^{N} \frac{\omega_{i} s_{i}}{N}=I_{\mathrm{S}} S
$$

where $S$ is the sensitivity of the index as a whole which is the weighted mean of the station sensitivities, $s_{i}$. To determine the station sensitivities $s_{i}$ from their known ratios we can either use a reference station (as Potsdam is used in the compilation of the $k p$ and $a p$ indices) or we can refer the data from each station $g_{i}$ to the overall index value $G$ and solve iteratively to generate a model of the index sensitivity $S(F, U T, G)$ (the method used by Lockwood et al., 2019d).

Figures $14 \mathrm{a}$ and $14 \mathrm{~b}$ show the index sensitivities, as computed by Lockwood et al. (2019d) for (a) am equal to its overall average value for all data taken to date $\left(\langle a m\rangle_{\text {all }}=\right.$ $21 \mathrm{nT}$ ) and (b) a high activity level, chosen here to be am above its $90 \%$ quantile of $q(0.9)=44 \mathrm{nT}$. The black, red and blue lines are for the $a m$, an and as indices (the global, northern hemisphere and southern hemisphere indices, respectively). The sensitivity variations with $U T$ are larger for all three indices (up to about 6\%) for the average activity level, but they are small (below 1\%) for the higher activity level. Figure $14 \mathrm{c}$ shows the variations with $U T$ (averaged over all $F$ and all years of their availability which is 1959-2017) am, an and as. Figure 14d shows the corresponding occurrence frequencies of the indices exceeding their 90 percentile value, $f[a m>q(0.9)], f[a n>q$ $(0.9)]$, and $f[a s>q(0.9)]$. In Figures $14 \mathrm{e}$ and $14 \mathrm{f}$ we have made allowances for the index response sensitivity by dividing the index by the relevant modelled sensitivity so the black red and blue lines are $a m / S_{\mathrm{am}}, a n / S_{\mathrm{an}}$, and $a s / S_{\mathrm{as}}$ in $14 \mathrm{e}$ and $f[a m /$ $\left.S_{\mathrm{am}}>q(0.9)\right], f\left[a n / S_{\mathrm{an}}>q(0.9)\right]$ and $f\left[a s / S_{\mathrm{as}}>q(0.9)\right]$ in $14 \mathrm{f}$. It can be see that allowance for sensitivity has made the $U T$ variations for the three indices much more similar for both the mean am and for the high-activity occurrence frequencies. The variations for average conditions, seen in (e), are small: for $a m$ the maximum and minimum values of the normalised $\langle a m\rangle / S_{\text {am }}$ are 1.04 and 0.93 (for 21-24 UT and 3-6 UT, respectively), giving and approximately $10 \%$ variation (peak to peak). For the high-activity event occurrence in $14 \mathrm{f}$, the $U T$ variations are slightly more pronounced: the maximum and minimum of the normalised $f\left[a m / S_{\mathrm{am}}>q(0.9)\right]$ are 1.10 and 0.89 (at the same $U T$ as for the $\langle a m\rangle / S_{\text {am }}$ variation) giving a $22 \%$ variation. In both $14 \mathrm{e}$ and $14 \mathrm{f}$ the minimum for an is at slightly earlier $U T$ than that for as. The $U T$-year spectrograms of $\langle a m\rangle / S_{\mathrm{am}}$ and $f$ $\left[a m / S_{\mathrm{am}}>q(0.9)\right]$ shown in parts $14 \mathrm{~g}$ and $14 \mathrm{~h}$ show that these minima are persistent from year-to-year. There is considerable speckling in these plots because of the random nature of the $U T$ of arrival of transient events hitting the Earth, particularly for the large event occurrence in $14 \mathrm{~h}$. However, a minimum at 03-06 UT is persistently present in both average and high activity event occurrence plots.

This analysis clearly demonstrates that the $U T$ variation in the am index is a real feature of geomagnetic activity and not caused by a response inhomogeneity due to the distribution of stations. Allowing for the index sensitivities has made the $U T$ variations in am, an and as more consistent but has not eliminated them. 


\section{Conclusions}

We have reviewed and investigated variations in geomagnetic activity with time of year, $F$ and Universal Time $U T$, by exploiting the exceptionally uniform $F-U T$ response of the am index (see Lockwood et al., 2019d). Other geomagnetic indices that do not have such a uniform $F-U T$ response, or which quantify effects that depend on integrated solar wind forcing, tend not to show the same F-UT activity pattern, although it can be identified in some variations derived from them. We have shown that variability in southward field normal to the equator equatorial plane (in the GSEQ frame) contributes over twice as much to the variability in the power input to the magnetosphere as does the Russell-McPherron (R-M) effect but, nevertheless, the semi-annual variation is almost entirely due to the R-M effect. One, or even both of the equinoctial peaks of the semiannual variation can be enhanced by chance because of the quasi-random nature of transient events hitting Earth, but this chance element is equally likely to reduce the semi-annual variation in any one year if more geoeffective events happen to strike Earth around the solstices.

We have compared the semi-annual variations in various geomagnetic indices to that seen simultaneously in the power input to the magnetosphere estimated from interplanetary data for the interval of near continuous IMF sampling (between 1995 and 2017, inclusive). Almost all show the same waveform with a larger September equinox peak. Analysis of the 150-year $a a_{\mathrm{H}}$ index sequence shows no clear pattern in which equinox is favoured and it appears to be a matter of chance set by at which equinox a greater number of more-geoeffective CMEs hit the Earth. However, the amplification of the waveform is different for each index and varies between just over 1 for $a \sigma$-noon to 10 for Dst. Mursula \& Karinen (2005) have discussed the large amplitude of the semi-annual variation in Dst and note that it arises from non-storm periods because of the way Dst is constructed. This is consistent with Figure 6 of the present paper that shows that although the occurrence of the top $10 \%$ of Dst values has a semiannual variation that is roughly twice the amplitude of the corresponding variation for $\mathrm{am}$, when we look at the top $1 \%$ of values we find $D s t$ and am have very similar semi-annual variations. Mursula \& Karinen (2005) suggest a correction that removes this tendency and make the response more dominated by storm effects. Lockwood et al. (2016) have shown that the largest $D s t$ values arise from long-lived large southward IMF in the GSE or GSEQ frames (often ahead or inside CMEs that intersect the Earth) and it is that which gives large southward IMF in GSM frame and an axial-like behaviour.

These points are emphasised by Figure 15. These panels show the relative influence of half-wave rectified southward IMF in the GSEQ frame ([$\left.B_{\mathrm{S}}\right]_{\mathrm{GSEQ}}$, the $x$ axis of each plot) and of the R-M effect, quantified by the difference between the half-wave rectified southward IMF in the GSM and GSEQ frames $\left(\left[B_{\mathrm{S}}\right]_{\mathrm{GSM}}-\left[B_{\mathrm{S}}\right]_{\mathrm{GSEQ}}\right.$, the $y$ axis). When $\left(\left[B_{\mathrm{S}}\right]_{\mathrm{GSM}}-\right.$ $\left.\left[B_{\mathrm{S}}\right]_{\mathrm{GSEQ}}\right)>0$ the RM effect is adding to the geo-effectiveness of the IMF, when $\left(\left[B_{\mathrm{S}}\right]_{\mathrm{GSM}}-\left[B_{\mathrm{S}}\right]_{\mathrm{GSEQ}}\right)<0$ the RM effect is reducing it. Figure 15a shows the number of samples in each bin on a logarithmic scale. (Note only bins containing more than 5 samples are shown). Figure $15 \mathrm{~b}$ shows the normalised power into the magnetosphere $P_{\alpha} / P_{o}$. It can be seen, as expected, mean

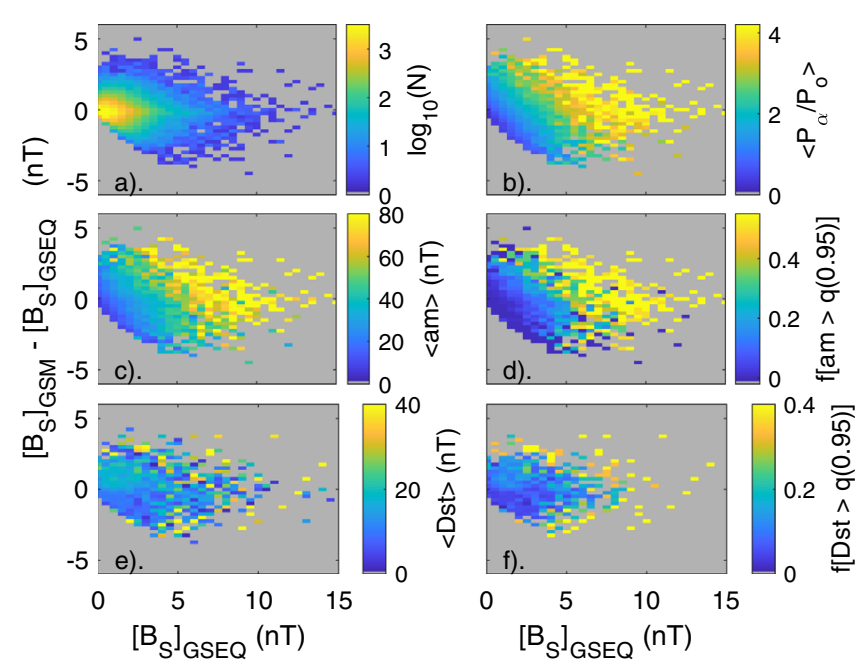

Figure 15. Analysis of the relative influences of large southward IMF in the GSEQ frame and of the R-M effect. In each panel the $x$-axis is $\left[B_{\mathrm{S}}\right]_{\mathrm{GSEQ}}$, as defined by equation (4) and data are binned into 30 bins of width $0.5 \mathrm{nT}$ between 0 and $15 \mathrm{nT}$. The R-M effect is quantified by how much the southward field in the GSM frame is enhanced over $\left[B_{\mathrm{S}}\right]_{\mathrm{GSEQ}}$, i.e. $\left(\left[B_{\mathrm{S}}\right]_{\mathrm{GSM}}-\left[B_{\mathrm{S}}\right]_{\mathrm{GSEQ}}\right)$ which is the $y$ axis in each panel and the data are sorted into 24 bins of bins of width $0.25 \mathrm{nT}$ between $-6 \mathrm{nT}$ and $+6 \mathrm{nT}$. The panels show for each bin (a) the logarithm of the number of samples, $N$, (b) the mean normalized power into the magnetosphere $\left\langle P_{\alpha} / P_{o}\right\rangle$; (c) the mean am index $\langle a m\rangle$; (d) the fraction am samples that exceed the $95 \%$ quantile, $f[a m>q(0.95)]$; (e) the mean of the $D s t$ index $\langle D s t\rangle$; (f) the fraction $D s t$ samples that exceed the $95 \%$ quantile, $f[D s t>q(0.95)]$. Note all data used in parts (a)-(d) are 3-hourly means, but the IMF averaging intervals have been shifted $1 \mathrm{~h}$ forward relative to the 3-hour am intervls to allow for the optimum am reponse lag. In parts (e) and (f) the Dst data are again 3-hourly means, but the IMF data are means over the previous $12 \mathrm{~h}$ which gives the optimum correlation (Lockwood et al., 2016). These plots for Dst were also made using the same three-hourly IMF averaging intervals used for am in parts (c) and (d) and the results were essentially the same (not shown). In all panels only values for bins containing more than five samples are shown. Data are for 1995-2017, inclusive.

values increase with both $\left[B_{\mathrm{S}}\right]_{\mathrm{GSEQ}}$ (to the right) and increased $\mathrm{R}-\mathrm{M}$ effect (up the page). Note that for $\left[B_{\mathrm{S}}\right]_{\mathrm{GSEQ}} \approx 0$ (the original R-M postulate), the R-M effect only raises $P_{\alpha} / P_{o}$ to average values that are less than half of those seen at large $\left[B_{\mathrm{S}}\right]_{\mathrm{GSEQ}}$, as noted from Figure 10 . Figure $15 \mathrm{c}$ shows that the average am response mirrors that of $P_{\alpha} / P_{o}$, as does the occurrence of large events in am, quantified by the occurrence frequency of am exceeding its 95th percentile, $f[a m>q(0.95)]$. The largest values of $P_{\alpha} / P_{o}, a m$, and $f[a m>q(0.95)]$ all occur at large $\left[B_{\mathrm{S}}\right]_{\mathrm{GSEQ}}$ with little or no assistance from the R-M effect (and sometimes despite some negative R-M effect). Essentially, the largest events are driven by CME events and CIRs crossings which deflect the IMF out of the solar equatorial plane in a southward direction and not by the R-M effect.

The behaviour of $D s t$ is interestingly different. In Figures $15 \mathrm{e}$ and $15 \mathrm{f}$ we have sorted the IMF data using the mean over the previous $12 \mathrm{~h}$, as Lockwood et al. (2016) found that gave the best correlation; however, results using the same 
procedure as for am yielded essentially identical results. The trend to larger means and more large events with increased $\left[B_{\mathrm{S}}\right]_{\mathrm{GSEQ}}$ can be seen in $15 \mathrm{e}$ and $15 \mathrm{f}$ but there is much more scatter than for am. The R-M effect is not detectable at large $\left[B_{\mathrm{S}}\right]_{\mathrm{GSEQ}}$, but is very clear at low $\left[B_{\mathrm{S}}\right]_{\mathrm{GSEQ}}$. This confirms the conclusion of Mursula \& Karinen (2005) that the large semiannual variation in Dst arises at small disturbance levels and not because of the major storms. We here confirm that semiannual variation in small and moderate $D s t$ values is driven by the R-M effect, but large storms are driven by sourhward field normal to the solar equatorial plane. This is not what one expects from the R-M paradigm: the original paper Russell \& McPherron (1973) specifically invokes the dipole tilt mechanism and IMF in the solar equatorial plane as the driver of large Dst storms. Figures $15 \mathrm{e}$ and $15 \mathrm{f}$ demonstrate that this is not the case, but large southward field normal to the solar equatorial plane is the driver. Yet there is no obvious reason why the occurrence of this large $\left[B_{\mathrm{S}}\right]_{\mathrm{GSEQ}}$ should yield the observed semi-annual variation in occurrence of large Dst storms at the equinoxes. However, we note that Figures $15 \mathrm{e}$ and $15 \mathrm{f}$ show a very strong R-M effect at low $\left[B_{\mathrm{S}}\right]_{\mathrm{GSEQ}}$ and we suggest that one possible mechanism is through the "pre-priming" of the magnetosphere ahead of large storms (see discussion by Lockwood et al., 2016). This is a potential solution of the paradox because enhanced pre-priming by the R-M effect (giving more small and moderate Dst disturbances) could cause the enhanced occurrence of major storms at the equinox even though those storms are driven by the impact of large $\left[B_{\mathrm{S}}\right]_{\mathrm{GSEQ}}$. An alternative explanation would be a second mechanism, in addition to the R-M effect, which enhances the largest storms at the equinoxes, and later papers will investigate the squeezing of the tail by solar wind dynamic pressure in this context.

We note that recently Poblet \& Azpilicueta (2018) have also defined the near-equinox peaks of a semi-annual variation in energetic electrons $(30 \mathrm{keV}-20 \mathrm{MeV})$ at L-shell values between 2.5 and 6.5, as observed by the Van Allen Probes. In addition Poblet et al. (2019) have found the semi-annual variation in PC5 wave intensity that can accelerate electrons to relativistic energies. Comparisons of the amplitudes and $F$-values of the peaks with other semianual variations (importantly for the same intervals of study) will be presented in a later paper.

This paper has concentrated on annual and semiannual variations in the solar wind drivers of geomagnetic activity. The energy, mass and momentum extracted from the solar wind has effects throughout the coupled magnetosphere-ionospherethermosphere system. However, we must also remember that the neutral thermosphere is not just a passive load where solar wind energy and particles are deposited (Sarris, 2019): it can have feedback effects on the ionosphere and magnetosphere that mean that there are non-linear responses to solar wind energy input, such as modulation of field-aligned current magnitudes and other effects of ionospheric conductivity distribution on electrodynamics (e.g. Lu et al., 1995). In addition, thermospheric wind, density and composition can modulate the composition of ion upflows into the inner magnetosphere (e.g. Yue et al., 2019). The thermosphere may also introduce additional variations through phenomena such as tides, planetary waves, atmospheric upwelling of heavier atoms/molecules and energy (e.g., Yamazaki et al., 2016) and the (varying and hemispherically asymmetric) offsets of the geomagnetic and rotational poles (Cnossen \& Richmond, 2012). These effects may influence annual and semiannual variations. A good illustration of the potential of such effects is provided by the equatorial electrojet ionospheric current which is known to be influenced by lunar tides to a greater extent during Northern Hemisphere winters and recent evidence suggests that stratospheric sudden warming events are a major part of the causal mechanism (e.g. Siddiqui et al., 2015). Hence we should not be surprised by non-linear responses to solar wind forcing and independent variations arising from lower atmospheric influences. Although we have not included any such effects in our analysis, by defining the solar-wind magnetosphere input as accurately as possible we hope to aid their detection.

In later papers in this series, we will investigate the amplification in geomagnetic data of the semi-annual variation in power input to the magnetosphere that is caused by the Russell-McPherron effect. In particular, we will highlight the key and separate role of solar wind dynamic pressure in this amplification, using an empirical model of the magnetopause and a global MHD model of the magnetosphere to demonstrate the effect. These considerations have to allow for the fact that the magnetosphere is rarely in a steady sate and substorms cycles occur (Lockwood et al., 1990; Cowley and Lockwood, 1992). We will also use Cluster observations of the near-Earth lobe field to confirm the mechanism.

Acknowledgements. The authors are grateful to the staff of the International Service of Geomagnetic Indices (ISGI), France and collaborating institutes for the compilation and databasing of the $a m$ and $a \sigma$ indices which were downloaded from http:// isgi.unistra.fr/data_download.php and to the staff of the Space Physics Data Facility (SPDF) at NASA's Goddard Space Flight Center for the Omni composite of interplanetary observations (made available by SPDF from https://omniweb.gsfc. nasa.gov/ow_min.html). For the SuperMAG indices data we gratefully acknowledge the PIs and staff of the many groups contributing data. SuperMAG data are available from http:// supermag.jhuapl.edu/indices/?layers=SME.UL. The homogeneous $a a$ index, $a a_{\mathrm{H}}$, is available as 3-hourly value or daily means from http://www.personal.reading.ac.uk/ ym901336/ pdfs/361_Lockwood2_SupplementaryMaterial_newaa_3hourly. txt. We are also grateful to the World Data Center for Geomagnetism, Kyoto for generating and making available the $A L, A U$ and Dst indices. (http://wdc.kugi.kyoto-u.ac.jp/).

This work is supported by a number of grants. MJO, CJS and ML at the University of Reading are supported by STFC consolidated grant number ST/M000885/1. The work of ML, LAB and MJO at University of Reading is also supported by the SWIGS NERC Directed Highlight Topic Grant number NE/P016928/1/. The work of JCC at the University of Southampton is supported by the UK Natural Environment Research Council (NERC) grant number NE/L007177/1 and by Science and Technology Facilities Council (STFC) Ernest Rutherford grant ST/L002809/1 and Consolidated grant ST/R000719/1. Funding for KAW at University of Saskatchewan was provided by the Canadian Foundation for Innovation (CFI), the Province of Saskatchewan, and a Discovery Grant from the Natural Sciences and Engineering Research 
Council (NSERC) of Canada. Initial work by KAW for this paper was carried out at University of Reading on sabbatical leave from University of Saskatchewan. $\mathrm{CH}$ is supported on a NERC PhD studentship as part of the SCENARIO Doctoral Training Partnership. The editor thanks Hermann Opgenoorth and an anonymous reviewer for their assistance in evaluating this paper.

\section{References}

Ahn BH, Kroehl HW, Kamide Y, Kihn E. 2000. Universal time variations of the auroral electrojet indices. J Geophys Res 105: 267-275. https://doi.org/10.1029/1999JA900364.

Ahn BH, Moon G-H. 2003. Seasonal and universal time variations of the AU, AL and Dst indices. J Korean Ast Soc 36: S93-S99.

Alexeev II, Belenkaya ES, Kalegaev VV, Feldstein Y-I, Grafe A. 1996. Magnetic storms and magnetotail currents. J Geophys Res 101: 7737-7747. https://doi.org/10.1029/95JA03509.

Allen JH, Kroehl HW. 1975. Spatial and temporal distributions of magnetic effects of auroral electrojets as derived from $\mathrm{AE}$ indices. J Geophys Res 80: 3667-3677. https://doi.org/10.1029/ JA080i025p03667.

Bartels J. 1925. Eine universelle tagsperiode der erdmagnetischen aktivität. Meteorol Z 42: 147.

Bartels J. 1949. The standardized index Ks and the planetary index Kp. IATME Bull 12: 97.

Boller BR, Stolov HL. 1970. Kelvin-Helmholtz instability and the semiannual variation of geomagnetic activity. J Geophys Res $\mathbf{7 5}$ : 6073. https://doi.org/10.1029/JA075i031p06073.

Broun JA. 1848. Observations in magnetism and meteorology made at Makerstoun in Scotland. Trans R Soc Edinburgh 18: 401-402.

Chapman S, Bartels J. 1940. Geomagnetism, Vol. II: Analysis of the Data, and Physical Theories. Oxford Univ. Press, London.

Caan MN, McPherron RL, Russell CT. 1973. Solar wind and substorm-related changes in the lobes of the geomagnetic tail. $J$ Geophys Res 78(34): 8087-8096. https://doi.org/10.1029/ ja078i034p08087.

Chambodut A, Marchaudon A, Menvielle M, El-Lemdani F, Lathuillere C. 2013. The K-derived MLT sector geomagnetic indices. Geophys Res Lett 40: 4808-4812. https://doi.org/10.1002/ grl.50947.

Chandler MO, Fuselier SA, Lockwood M, Moore TE. 1999. Evidence of component magnetic merging equatorward of the cusp. J Geophys Res 104: 22623-22648. https://doi.org/10.1029/ 1999JA900175.

Chu X, McPherron RL, Hsu T-S, Angelopoulos V. 2015. Solar cycle dependence of substorm occurrence and duration: Implications for onset. J Geophys Res Space Phys 120: 2808-2818. https://doi.org/ 10.1002/2015JA021104.

Clauer CR, McPherron RL. 1974. Mapping the local time-universal time development of magnetospheric substorms using mid-latitude magnetic observations. J Geophys Res 79(19): 2811-2820. https:// doi.org/10.1029/JA079i019p02811.

Cliver EW, Kamide Y, Ling AG. 2000. Mountains versus valleys: Semiannual variation of geomagnetic activity. J Geophys Res 105: 2413-2424. https://doi.org/10.1029/1999JA900439.

Cliver EW, Kamide Y, Ling AG. 2002. The semiannual variation of geomagnetic activity: phases and profiles for 130 years of aa data. J Atmos Sol Terr Phys 64: 47-53. https://doi.org/10.1016/s13646826(01)00093-1.
Cnossen I, Richmond AD. 2012. How changes in the tilt angle of the geomagnetic dipole affect the coupled magnetosphere-ionospherethermosphere system. J Geophys Res 117: A10317. https://doi.org/ 10.1029/2012JA018056.

Cortie AL. 1912. Sunspots and terrestrial magnetic phenomena, 1898-1911. Mon Not Roy Astron Soc 73: 52-60. https://doi.org/ 10.1093/mnras/73.1.52.

Cowley SWH, Lockwood M. 1992. Excitation and decay of solarwind driven flows in the magnetosphere-ionosphere system. Ann Geophys 10: 103-115.

Crooker NU, Siscoe GL. 1986. On the limits of energy transfer through dayside merging. J Geophys Res 91: 13393-13397. https://doi.org/10.1029/JA091iA12p13393.

Danilov AA, Krymskii GF, Makarov GA. 2013. Geomagnetic activity as a reflection of processes in the magnetospheric tail: 1 . The source of diurnal and semiannual variations in geomagnetic activity. Geomag. Aeron. 53: 441-447. https://doi.org/10.1134/S0016793213040051.

Davis TN, Sugiura M. 1966. Auroral electrojet activity index AE and its universal time variations. J Geophys Res 71(3): 785-801. https://doi.org/10.1029/JZ071i003p00785.

de La Sayette P, Berthelier A. 1996. The am annual-diurnal variations 1959-1988: A 30-year evaluation. J Geophys Res 101(A5): 10653-10663. https://doi.org/10.1029/96JA00165.

de La Sayette P. 2004. Empirical simulations for the am annualdiurnal activity. J Geophys Res 109: A07207. https://doi.org/ 10.1029/2003JA010353.

Ebert RW, McComas DJ, Elliott HA, Forsyth RJ, Gosling JT. 2009. Bulk properties of the slow and fast solar wind and interplanetary coronal mass ejections measured by Ulysses: Three polar orbits of observations. J Geophys Res 114: A01109. https://doi.org/ 10.1029/2008JA013631.

Finch ID. 2008. The use of geomagnetic activity observations in studies of solar wind-magnetosphere coupling and centennial solar change, PhD thesis, Southampton University, Southampton, UK.

Finch ID, Lockwood M. 2007. Solar wind-magnetosphere coupling functions on timescales of 1 day to 1 year. Ann Geophys 25: 495506. https://doi.org/10.5194/angeo-25-495-2007.

Finch ID, Lockwood M, Rouillard AP. 2008. The effects of solar wind magnetosphere coupling recorded at different geomagnetic latitudes: separation of directly-driven and storage/release systems. Geophys Res Lett 35: L21105. https://doi.org/10.1029/2008GL035399.

Hajkowicz LA. 1992. Universal time effect in the occurrence of large-scale ionospheric disturbances. Planet Space Sci 40: 10931099. https://doi.org/10.1016/0032-0633(92)90038-p.

Hajkowicz LA. 1998. Longitudinal (UT) effect in the onset of auroral disturbances over two solar cycles as deduced from the AE-index. Ann Geophys 16(12): 1573-1579. https://doi.org/10.1007/s00585998-1573-9.

Hundhausen AJ, Bame SJ, Montgomery MD. 1971. Variations of solar-wind plasma properties: Vela observations of a possible heliographic latitude-dependence. J Geophys Res 76: 5145-5154. https://doi.org/10.1029/ja076i022p05145.

Karlsson SBP, Opgenoorth HJ, Eglitis P, Kauristie K, Syrjäsuo M, Pulkkinen TI, Lockwood M, Nakamura R, Reeves G, Romanov S. 2000. Solar wind control of magnetospheric energy content: substorm quenching and multiple onsets. J Geophys Res 105: 5335-5356. https://doi.org/10.1029/1999JA900297.

Kivelson MG, Hughes WJ. 1990. On the threshold for triggering substorms. Planet Space Sci 38: 211-220. https://doi.org/10.1016/ 0032-0633(90)90085-5.

Klimenko MV, Klimenko VV, Bryukhanov VV. 2007. Numerical modeling of the equatorial electrojet UT-variation on the basis of the model GSM TIP. Adv Radio Sci 5: 385-392. 
Kokubun S, McPherron RL, Russell CT. 1977. Triggering of substorms by solar wind discontinuities. J Geophys Res 82(1): 74-86. https://doi.org/10.1029/ja082i001p00074.

Kubyshkina M, Tsyganenko N, Semenov V, Kubyshkina D, Partamies N, Gordeev E. 2015. Further evidence for the role of magnetotail current shape in substorm initiation. Earth Planets Space 67: 139. https://doi.org/10.1186/s40623-015-0304-1.

Kuznetsova TV, Laptukhov AI. 2011. Contribution of geometry of interaction between interplanetary and terrestrial magnetic fields into global magnetospheric state and geomagnetic activity. $A d v$ Space Res 47: 978-990. https://doi.org/10.1016/j.asr.2010.11.022.

Le Mouël J-L, Blanter E, Chulliat A, Shnirman M. 2004. On the semiannual and annual variations of geomagnetic activity and components. Ann Geophys 22: 3583-3588. https://doi.org/ 10.5194/angeo-22-3583-2004.

Lockwood M. 2013. Reconstruction and Prediction of Variations in the Open Solar Magnetic Flux and Interplanetary Conditions. Living Rev Sol Phys 10(4): 2013. https://doi.org/10.12942/lrsp-2013-4.

Lockwood M. 2019. Does adding solar wind Poynting flux improve the optimum solar wind - magnetosphere coupling function? $J$ Geophys Res Space Phys 124(7): 5498-5515. https://doi.org/ 10.1029/2019JA026639.

Lockwood M, Cowley SWH, Freeman MP. 1990. The excitation of plasma convection in the high latitude ionosphere. J Geophys Res 95: 7961-7971. https://doi.org/10.1029/JA095iA06p07961.

Lockwood M, Owens MJ, Barnard LA, Bentley S, Scott CJ, Watt CE. 2016. On the origins and timescales of geoeffective IMF. Space Weather 14: 406-432. https://doi.org/10.1002/2016SW001375.

Lockwood M, Chambodut A, Barnard LA, Owens MJ, Clarke E, et al.. 2018a. A homogeneous aa index: 1. Secular variation. J Space Weather Space Clim 8: A53. https://doi.org/10.1051/swsc/2018038.

Lockwood M, Finch ID, Chambodut A, Barnard LA, Owens MJ, Clarke E. 2018b. A homogeneous aa index: 2. hemispheric asymmetries and the equinoctial variation. J Space Weather Space Clim 8: A58. https://doi.org/10.1051/swsc/2018044.

Lockwood M, Bentley S, Owens MJ, Barnard LA, Scott CJ, Watt CE, Allanson O. 2019a. The development of a space climatology: 1. Solar-wind magnetosphere coupling as a function of timescale and the effect of data gaps. Space Weather 17: 133-156. https:// doi.org/10.1029/2018SW001856.

Lockwood M, Bentley S, Owens MJ, Barnard LA, Scott CJ, Watt CE, Allanson O, Freeman MP. 2019b. The development of a space climatology: The distribution of power input into the magnetosphere on a 3-hourly timescale. Space Weather 17: 157-179. https://doi.org/10.1029/2018SW002016.

Lockwood M, Bentley S, Owens MJ, Barnard LA, Scott CJ, Watt CE, Allanson O, Freeman MP. 2019c. The development of a space climatology: 3. The evolution of distributions of space weather parameters with timescale. Space Weather 17: 180-209. https:// doi.org/10.1029/2018SW002017.

Lockwood M, Chambodut A, Finch ID, Barnard LA, Owens MJ, Haines C. 2019d. Time-of-day / time-of-year response functions of planetary geomagnetic indices. J Space Weather Space Clim 9: A20. https://doi.org/10.1051/swsc/2019017.

Lockwood M, Owens MJ, Macneil A. 2019e. On the origin of orthogardenhose heliospheric flux. Sol Phys 294: 58. https://doi.org/ 10.1007/s11207-019-1478-7.

Lu G, Richmond AD, Emery BA, Roble RG. 1995. Magnetosphereionosphere-thermosphere coupling: Effect of neutral winds on energy transfer and field-aligned current. J Geophys Res 100(A10): 19643-19659. https://doi.org/10.1029/95JA00766.

Lyatsky W, Newell PT, Hamza A. 2001. Solar illumination as the cause of the equinoctial preference for geomagnetic activity.
Geophys Res Lett 28(12): 2353-2356. https://doi.org/10.1029/ 2000GL012803.

Mayaud P-N. 1971. Une mesure planétaire d'activité magnetique, basée sur deux observatoires antipodaux. Ann Geophys 27: 67-70.

Mayaud P-N. 1972. The $a a$ indices: A 100-year series characterizing the magnetic activity. J Geophys Res 77: 6870-6874. https://doi. org/10.1029/JA077i034p06870.

Mayaud P-N. 1980. Derivation, Meaning and Use of Geomagnetic Indices. Geophysical Monograph 22, American Geophysical Union, Washington, DC. https://doi.org/10.1029/GM022.

McComas DJ, Ebert RW, Elliott HA, Goldstein BE, Gosling JT, Schwadron NA, Skoug RM. 2008. Weaker solar wind from the polar coronal holes and the whole Sun. Geophys Res Lett 35: L18103. https://doi.org/10.1029/2008GL034896.

McIntosh DH. 1959. On the annual variation of magnetic disturbances. Phil Trans Roy Soc London A 251(1001): 525552. https://doi.org/10.1098/rsta.1959.001.

McPherron RL, Baker DN, Pulkkinen TI, Hsu TS, Kissinger J, Chu X. 2013. Changes in solar wind-magnetosphere coupling with solar cycle, season, and time relative to stream interfaces. J Atmos Sol Terr Phys 99: 1-13. https://doi.org/10.1016/j.jastp.2012.09.003.

Menvielle M, Berthelier A. 1991. The K-derived planetary indices: Description and availability. Rev Geophys 29(3): 415-432. https:// doi.org/10.1029/91RG00994.

Mursula K, Karinen A. 2005. Explaining and correcting the excessive semiannual variation in the Dst index. Geophys Res Lett 32: L14107. https://doi.org/10.1029/2005GL023132.

Newell PT, Gjerloev JW. 2011a. Evaluation of SuperMAG auroral electrojet indices as indicators of substorms and auroral power. $J$ Geophys Res 116: A12211. https://doi.org/10.1029/2011JA016779.

Newell PT, Gjerloev JW. 2011b. Substorm and magnetosphere characteristic scales inferred from the SuperMAG auroral electrojet indices. J Geophys Res 116: A12232. https://doi.org/10.1029/ 2011JA016936.

Newell PT, Gjerloev JW. 2012. SuperMAG-based partial ring current indices. J Geophys Res 117: A05215. https://doi.org/ 10.1029/2012JA017586.

Newell PT, Sotirelis T, Skura JP, Meng C-I, Lyatsky W. 2002. Ultraviolet insolation drives seasonal and diurnal space weather variations. J Geophys Res 107(A10): 1305. https://doi.org/ 10.1029/2001JA000296.

Nowada M, Shue J-H, Russell CT. 2009. Effects of dipole tilt angle on geomagnetic activity. Planet Space Sci 57(11): 1254-1259. https://doi.org/10.1016/j.pss.2009.04.007.

O'Brien TP, McPherron RL. 2002. Seasonal and diurnal variation of Dst dynamics. J Geophys Res 107(A11): 1341. https://doi.org/ 10.1029/2002JA009435.

Olson WP. 1969. The shape of the tilted magnetopause. J Geophys Res 74: 5642-5651. https://doi.org/10.1029/JA074i024p05642.

Perreault P, Akasofu S-I. 1978. Study of Geomagnetic Storms. Geophys J Roy Astronom Soc 54(3): 547-573. https://doi.org/ 10.1111/J.1365-246x.1978.Tb05494.X.

Pinto O, Gonzalez WD. 1989. Energetic electron precipitation at the South Atlantic Magnetic Anomaly: a review. J Atmos Terr Phys 51 (5): 351-365. https://doi.org/10.1016/0021-9169(89)90117-7.

Poblet FL, Azpilicueta F. 2018. Semiannual variation in radiation belts particle fluxes: Van Allen probes observations. Ann Geophys Discuss 98: 1-18. https://doi.org/10.5194/angeo-2018-98.

Poblet FL, Azpilicueta F, Lam HL. 2019. Semiannual variation of Pc5 ULF waves and relativistic electrons over two solar cycles of observations: comparison with predictions of the classical hypotheses. Ann Geophys. Discuss 157: 1-24. https://doi.org/ 10.5194/angeo-2019-157. 
Rosenberg RL, Coleman PJ Jr. 1969. Heliographic latitude dependence of the dominant polarity of the interplanetary magnetic field. J Geophys Res 74: 5611-5622. https://doi.org/10.1029/ JA074i024p05611.

Russell CT. 1989. The universal time variation of geomagnetic activity. Geophys Res Lett 16(6): 555-558. https://doi.org/10.1029/ g1016i006p00555.

Russell CT, McPherron RL. 1973. Semiannual variation of geomagnetic activity. J Geophys Res 78: 82-108. https://doi.org/ 10.1029/JA078i001p00092.

Russell CT, Wang YL, Raeder J. 2003. Possible dipole tilt dependence of dayside magnetopause reconnection. Geophys Res Lett 30(18): 1937. https://doi.org/10.1029/2003GL017725.

Sabine E. 1852. On periodical laws discoverable in the mean effects of the larger magnetic disturbances, II. Philos Trans $R$ Soc London 142: 103-124. https://doi.org/10.1098/rstl.1852.0009.

Sarris TE. 2019. Understanding the ionosphere thermosphere response to solar and magnetospheric drivers: status, challenges and open issues. Philos Trans R Soc London 377: 20180101. https://doi.org/10.1098/rsta.2018.0101.

Schieldge JP, Siscoe GL. 1970. A correlation of the occurrence of simultaneous sudden magnetospheric compressions and geomagnetic bay onsets with selected geophysical indices. J Atmos Terr Phys 32(11): 1819-1830. https://doi.org/10.1016/0021-9169(70) 90139-x.

Sergeev VA, Tsyganenko NA, Smirnov MV, Nikolaev AV, Singer H, Baumjohann W. 2011. Magnetic effects of the substorm current wedge in a "spread-out wire" model and their comparison with ground, geosynchronous, and tail lobe data. J Geophys Res 116: A07218. https://doi.org/10.1029/2011JA016471.

Siddiqui TA, Lühr H, Stolle C, Park J. 2015. Relation between stratospheric sudden warming and the lunar effect on the equatorial electrojet based on Huancayo recordings. Ann Geophys 33: 235-243. https://doi.org/10.5194/angeo-33-235-2015.

Svalgaard L. 1977. Geomagnetic activity: Dependence on solar wind parameters. In Coronal Holes and High Speed Wind Streams. Zirker JB, (Ed.). Report SU-IPR-699, California Institute For Plasma Research, Stanford University, pp. 371-441. http://adsabs. harvard.edu/abs/1977chhs.conf..371S.

Takalo J, Mursula K. 2001. A model for the diurnal universal time variation of the Dst index. J Geophys Res 106(A6): 10905-10913. https://doi.org/10.1029/2000JA000231.

Tanskanen EI. 2009. A comprehensive high-throughput analysis of substorms observed by IMAGE magnetometer network: Years 1993-2003 examined. J Geophys Res 114: A05204. https://doi. org/10.1029/2008JA013682.

Thébault E, Finlay CC, Beggan CD, Alken P, Aubert J, et al. 2015. International Geomagnetic Reference Field: the 12th generation. Earth Planet Space 67: 79. https://doi.org/10.1186/s40623-0150228-9.

Vasyliunas VM, Kan JR, Siscoe GL, Akasofu S-I. 1982. Scaling relations governing magnetospheric energy transfer. Planet Space Sci 30: 359-365. https://doi.org/10.1016/0032-0633(82)90041-1.

Weigel RS. 2007. Solar wind time history contribution to the day-ofyear variation in geomagnetic activity. $J$ Geophys Res 112: A10207. https://doi.org/10.1029/2007JA012324.

Whang YC, Wang Y-M, Sheeley NR Jr, Burlaga LF. 2005. Global structure of the out-of-ecliptic solar wind. J Geophys Res 110: A03103. https://doi.org/10.1029/2004JA010875.

Yamazaki Y, Häusler K, Wild JA. 2016. Day-to-day variability of midlatitude ionospheric currents due to magnetospheric and lower atmospheric forcing. J Geophys Res Space Phys 121: 7067-7086. https://doi.org/10.1002/2016JA022817.
Yue C, Bortnik J, Li W, Ma Q, Wang C-P, Thorne RM, et al. 2019. Oxygen ion dynamics in the Earth's ring current: Van Allen Probes observations. J Geophys Res Space Phys 124: 7786-7798. https://doi.org/10.1029/2019JA026801.

Yue C, Zong QG, Zhang H, Wang YF, Yuan CJ, Pu ZY, Fu SY, Lui ATY, Yang B, Wang CR. 2010. Geomagnetic activity triggered by interplanetary shocks. J Geophys Res 115: A00I05. https://doi.org/ 10.1029/2010JA015356.

Zhao H, Zong QG. 2012. Seasonal and diurnal variation of geomagnetic activity: Russell-McPherron effect during different IMF polarity and/or extreme solar wind conditions. J Geophys Res 117: A11222. https://doi.org/10.1029/2012JA017845.

\section{Appendix A}

\section{Daily $A a_{H}$ values as an indicator of substorm activity}

The 3-hourly $a a_{\mathrm{H}}$ index and its daily means $A a_{\mathrm{H}}$ (like the corresponding am and Am indices) cannot detect individual substorm cycles which are of order an hour in duration. However, comparison with indices that are good detectors of substorms, for example the auroral electrojet $A E$ and $A L$ indices (Davis \& Sugiura, 1966) and their SuperMAG equivalents $S M E$ and $S M L$ (Newell \& Gjerloev, 2011a, b), show that they are strongly related to mid-latitude indices such as $a a_{\mathrm{H}}$ and $a m$ when averaged over the same intervals, as for example shown by Figure 3c of Lockwood et al. (2019d) and the supporting infromation file attached to Lockwood et al. (2019a).

This is further emphasised in this Appendix, using daily means of the $A E$ and $A L$ indices and comparing them to $A a_{\mathrm{H}}$ values measured over the interval 1995-2017, inclusive.

The $a a_{\mathrm{H}}$ index, like $a m$, is a "range" index, meaning that it is based on the difference between the maximum and minimum values of the horizontal field component in each 3-hourly interval. Hence, like all range indices, it will quantify of the largest disturbances that occur within the 3-hour interval and substorms are a phenomenon that generate large variations a mid-latitudes on timescales smaller than $3 \mathrm{~h}$ (e.g., Clauer \& McPherron, 1974; Sergeev et al., 2011). Hence we here compare to the maximum $A E\left(A E_{\max }\right)$ and minimum $A L\left(A L_{\min }\right)$ seen in the 3-hour intervals that $a a_{\mathrm{H}}$ is compiled over. (Remember that $A L$ is negative in substorms and that $A E=A U-A L$ ). The analysis was also carried for the mean values of $A E$ and $A L$ over the 3-hour intervals: the results were very similar but with slightly greater scatter.

Figure $\mathrm{A} 1$ shows scatter plots of $A E_{\max }$, the maximum value of the 1-minute $A E$ index in each $3 \mathrm{~h}$ interval over which an $a a_{\mathrm{H}}$ index value is derived. In the three panels of Figure A1 these data have been averaged over intervals $\tau$ of (a) 1 day; (b) 27 days (aproximately a solar rotation interval as seen from Earth) and (c) 1 year. The scatter in the relationship is reduced as averaging timescale $\tau$ is increased and is minimal for $\tau=1$ year when the seasonal variations in the $A E$ and $A L$ indices (which are compiled from northern hemisphere stations only) are averaged out. Averaging also supresses random observational noise. For the daily values (Fig. A1-a) we see a marked non-linearity on the higher-activity days with the ratio $A a_{\mathrm{H}} /<A E_{\max }>$ increasing at large values. This non-linearity is weaker but still present in the 27-day averages (Fig. A1-b) but is averaged out, giving a linear relationship, for averages over 1 year (Fig. A1-c). 


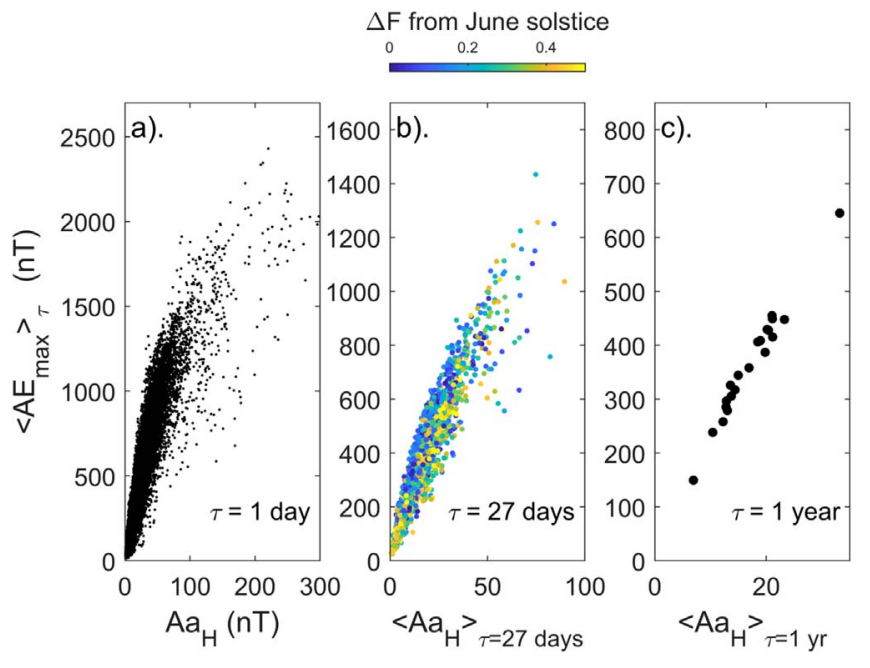

Figure A1. Scatter plots of the maximum value of the $A E$ auroral electrojet index in $3 \mathrm{~h}$ intervals, $A E_{\max }$, as a function of the homogeneous $a a$ index value, $a a_{\mathrm{H}}$, from the same 3-hour interval. Plots are for three averaging timescales $\tau$ of both data series: (a) $\tau=1$ day (note that $\left\langle a a_{\mathrm{H}}\right\rangle_{\tau}=1$ day is called $A a_{\mathrm{H}}$ ); (b) $\tau=27$ days and (c) $\tau=1$ year. Data are for 1995 to 2017 inclusive. In the middle panel, the data points have been colour-coded according to the difference in $F$ between the centre of the 27-day interval and the closest June solstice, $\Delta F$.
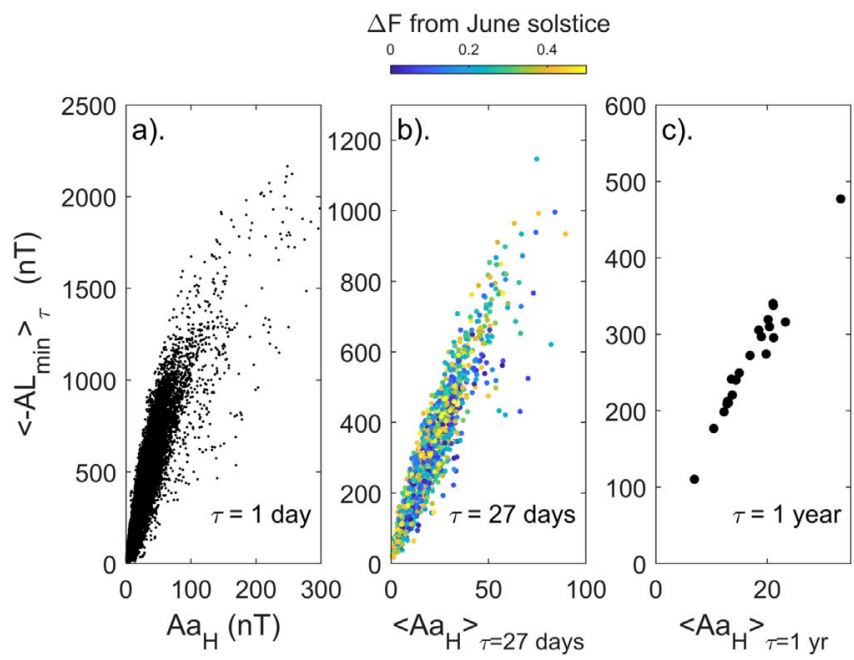

Figure A2. The same as Figure A1 for the negative of the minimum value of the $A L$ auroral electrojet index in $3 \mathrm{~h}$ intervals, $-A L_{\text {min }}$.

In part (b) the datapoints have been colour coded by the time-of-year of the centre of each 27-day interval, the colour scale being $\Delta F$, the separation in time to the closest June solstice. Hence blue points are close to summer solstice and yellow points are close to the winter solstice for the (northernhemisphere) $A E$ stations. Figure A1-b shows that for a given $\left\langle A a_{\mathrm{H}}\right\rangle_{\tau}=27$ days the corresponding $A E$ value tends to be largest at summer solstice and hence much of the rather small scatter that does exist is due to the seasonal dependence of $A E$. Note that this colour coding has not been applied to
Figure A1-a because it contains 61,362 data points and such high density of points means that they are overplotted to the extent that the colouration depends strongly on the order in which the points are plotted.

Figure $\mathrm{A} 2$ repeats the same plot for $-A L_{\mathrm{min}}$, the negative of the minimum value of the $A L$ index in the 3-hour interval in which each $a a_{\mathrm{H}}$ value is compiled. The behaviour is extremely similar showing it is the nightside auroral electrojet of the substorm current wedge that $a a_{\mathrm{H}}$, and all mid-latitude range indices, primarily responds to. However, Figure A2-b does not show the time of year effect that is evident in Figure A1-b: hence the seasonal dependence in $A E$ appears to mainly arise from $A U$ and not $A L$.

Very similar plots are obtained for the SuperMAG auroral eletrojet indices, $S M L$ and $S M E$. These indices are constructed with a resolution of 1 minute in the same way as the $A L$ and $A E$ auroral electrojet indices, but instead of taking the maximum and minimum detected by a ring of 12 northern-hemisphee auroral stations, $S M L$ and $S M E$ employ all avaliable stations in the northern hemisphere, which for the interval studied typically number over 100 (Newell \& Gjerloev, 2011a, b).

\section{Appendix B}

\section{The relative effects of southward field in the GSEQ frame and of the Russell-McPherron mechanism}

In this paper, we make use of two trigonometric functions in the GSM reference frame to quantify the IMF orientation factor control of solar wind geoeffectiveness. The first is the half-wave rectified southward component of the IMF in the GSM frame (for unit IMF magnitude in the $Y-Z$ plane), $\left[B_{S}\right]_{\mathrm{GSM}} / B_{Y Z}$, where $\left[B_{S}\right]_{\mathrm{GSM}}$ is defined by equation (4) of the text and employed in the initial paper on the R-M effect by Russell \& McPherron (1973). The second is $\sin ^{4}\left(\theta_{\mathrm{GSM}} / 2\right)$, where $\theta_{\mathrm{GSM}}$ is the IMF

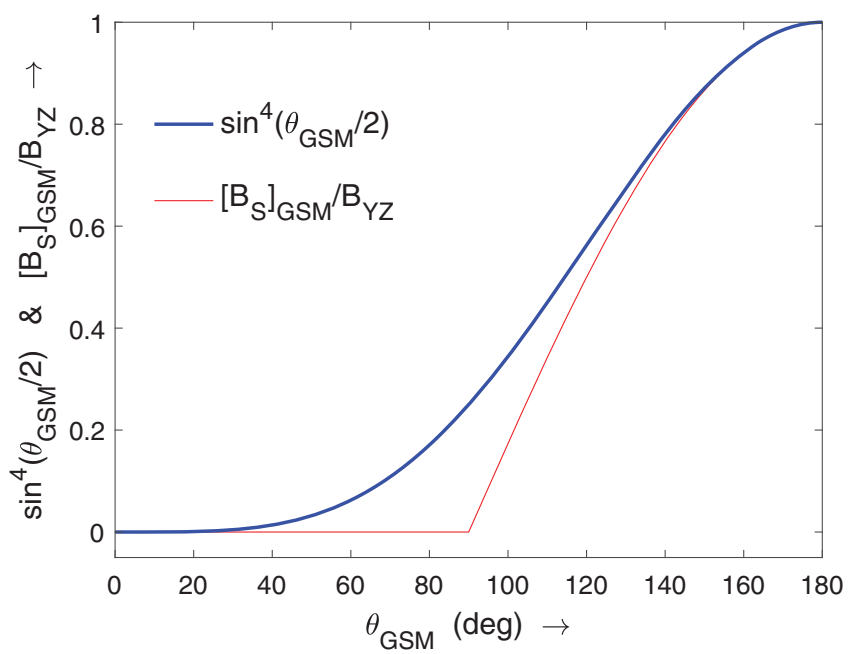

Figure B1. The two IMF orientation factors in solar-wind/magnetosphere coupling functions used in this paper: (blue) $\sin ^{4}\left(\theta_{\mathrm{GSM}} / 2\right)$, where $\theta_{\mathrm{GSM}}$ is the IMF clock angle in the GSM frame, and (red) $\left[B_{S}\right]_{\mathrm{GSM}} / B_{Y Z}$, where $\left[B_{S}\right]_{\mathrm{GSM}}$ is the halfwave-rectified southward field in the GSM frame and $B_{X Y}$ is the magnitude of the field in the $Y-Z$ plane (which is the same for the GSE, GSEQ and GSM frames). Both are plotted as a function of $\theta_{\mathrm{GSM}}$. 

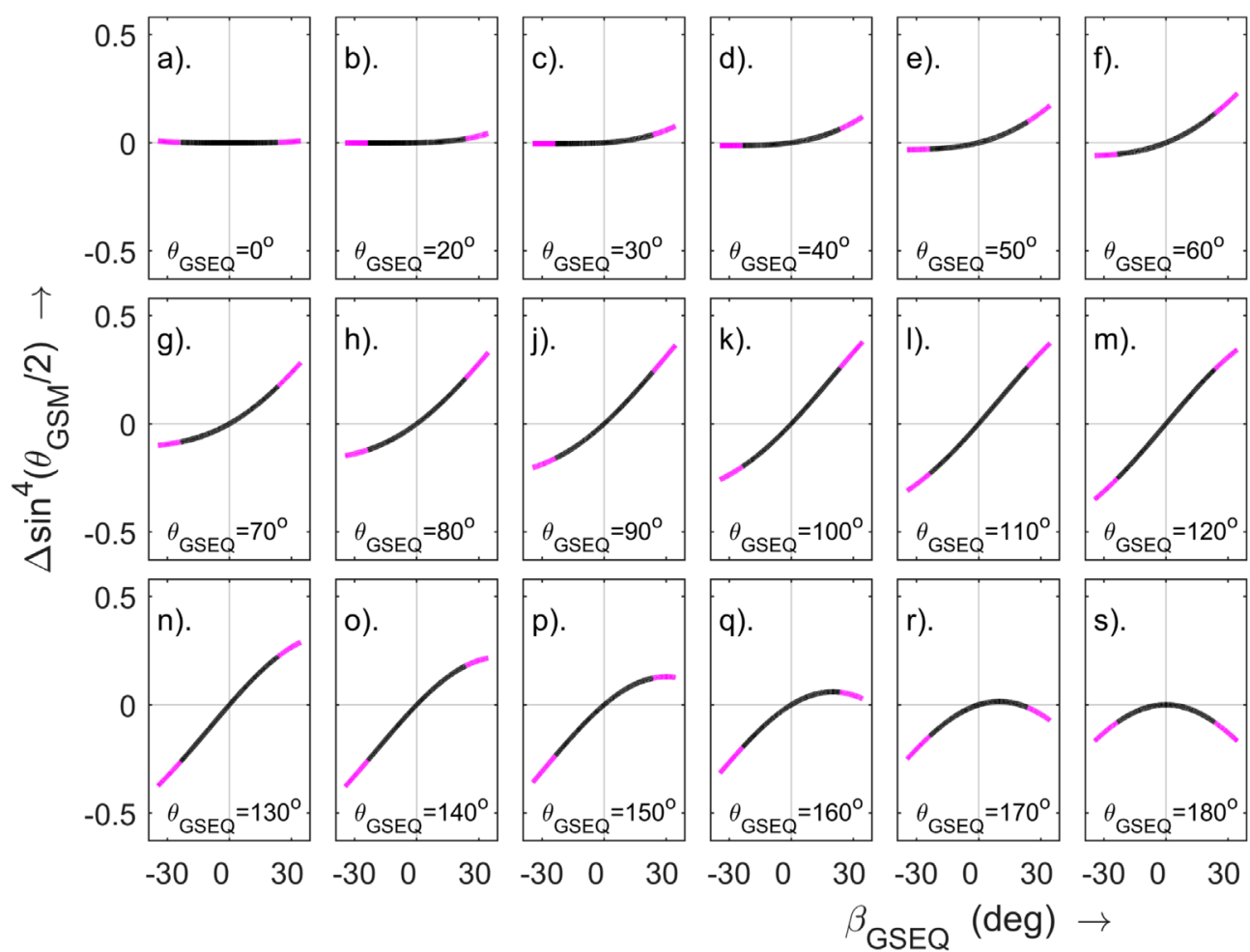

Figure B2. Variations in the $\sin ^{4}\left(\theta_{\mathrm{GSM}} / 2\right) \mathrm{IMF}$ orientation factor at a given $\beta_{\mathrm{GSEQ}}$ (the GSEQ-to-GSM frame rotation angle) and $\theta_{\mathrm{GSEQ}}$ (the IMF clock angle in the GSEQ frame). The plots are of $\Delta \sin ^{4}\left(\theta_{\mathrm{GSM}} / 2\right)$, the difference in the value for the $\beta_{\mathrm{GSEQ}}$ given by the $x$ axis and for $\beta_{\mathrm{GSEQ}}=0$ : hence $\Delta \sin ^{4}\left(\theta_{\mathrm{GSM}} / 2\right)$ quantifies the fractional variation in the solar-wind/magnetosphere coupling due to the R-M effect, positive values being increases. The mauve lines are for the $U T$ effect and the annual $(F)$ effects combined (due to the offset of Earth's magnetic and rotational axes and the tilt of Earth's rotational axis to the ecliptic plane, respectively) and the black segments are for the annual $(F)$ variation alone. Each panel is for a different value of $\theta_{\mathrm{GSEQ}}$ between 0 (purely northward IMF normal to the solar equator) and $180^{\circ}$ (purely southward IMF normal to the solar equator).

clock angle in the GSM frame, as used in both the $P_{\alpha}$ and $\varepsilon$ solar-wind/magnetosphere coupling functions defined in equations (2) and (3) of the main text. Figure B1 plots the variations of the two as a function of $\theta_{\mathrm{GSM}}$. It can be seen that $\sin ^{4}\left(\theta_{\mathrm{GSM}} / 2\right)$ does not have the disadvantage of an unphysical discontinuous change of slope at $\theta_{\mathrm{GSM}}=90^{\circ}$ and allows some coupling at weakly northward IMF ( $\theta_{\mathrm{GSM}}$ approaching, but less than, $90^{\circ}$ ) through reconnection in the dayside magnetopause. Studies show that $\sin ^{4}\left(\theta_{\mathrm{GSM}} / 2\right)$ provides an optimum match to the observations (Lockwood et al., 2018a, b; Lockwood, 2019).

We here consider the effect of dipole tilt (R-M) and out-ofequator field, $\left[B_{S}\right]_{\mathrm{GSEQ}}$ on these coupling functions. The dipole tilt (R-M) effect in Figure B2 is for $\sin ^{4}\left(\theta_{\mathrm{GSM}} / 2\right)$ and in Figure $\mathrm{B} 3$ is for $\left[B_{S}\right]_{\mathrm{GSM}} / B_{Y Z}$. In both cases, we plot the variations due to the R-M effect by studying the full range of the GSEQ to GSM rotation angles $\beta_{\mathrm{GSEQ}}$. The mauve lines are for the $U T$ effect and the annual $(F)$ variation combined (due to the offset of Earth's magnetic and rotational axes and the tilt of Earth's rotational axis to the ecliptic plane, respectively) and the black segments are for the annual $(F)$ variation alone. Each plot show the difference in the coupling function with respect to that for $\beta_{\mathrm{GSEQ}}=0$ (because at $\beta_{\mathrm{GSEQ}}=0$ the R-M mechanism has no effect), namely $\Delta \sin ^{4}\left(\theta_{\mathrm{GSM}} / 2\right)$ in Figure B2 and $\Delta\left[B_{S}\right]_{\mathrm{GSM}} /$ $B_{X Y}$ in Figure B3. Each panel is for a different value of $\theta_{\mathrm{GSEQ}}$, the IMF clock angle in the GSEQ frame, such that $\theta_{\mathrm{GSEQ}}=0$ is for purely northward IMF, normal to the solar equator; $\theta_{\mathrm{GSEQ}}$ $=180^{\circ}$ is for purely southward IMF normal to the solar equator; and $\theta_{\mathrm{GSEQ}}=90^{\circ}$ is for IMF in the solar equatorial plane (the orientation used by Russell \& McPherron (1973) to describe the concept of the R-M effect). (Note that the $\beta_{\mathrm{GSEQ}}=10^{\circ}$ plots are omitted to keep the number of panels to 18 and the results are almost identical to those for the $\beta_{\mathrm{GSEQ}}=0$ ).

In interpreting Figures $\mathrm{B} 2$ and $\mathrm{B} 3$ it should be remembered that positive values of $\Delta \sin ^{4}\left(\theta_{\mathrm{GSM}} / 2\right)$ and $\Delta\left[B_{S}\right]_{\mathrm{GSM}} / B_{Y Z}$ (respectively) mean that the R-M effect is adding to pre-existing southward field in the GSEQ frame (i.e., making the IMF more geo-effective) and negative values mean that the R-M effect is subtracting from it (i.e., making it less geo-effective).

The bottom rows of Figures B2 and B3 (panels n-s) show that for both the coupling functions, large southward IMF in the GSEQ frame $\left(\theta_{\mathrm{GSEQ}} \geq 130^{\circ}\right)$ the net effect of the R-M effect is to reduce geoeffectiveness because the net gain in geoeffective IMF for $\beta_{\mathrm{GSEQ}}>0$ is smaller than the net reduction for $\beta_{\mathrm{GSEQ}}<0$. This asymmetry grows with increased $\theta_{\mathrm{GSEQ}}$ until for $\theta_{\mathrm{GSEQ}}=180^{\circ}$ (panel s, purely southward IMF in the 

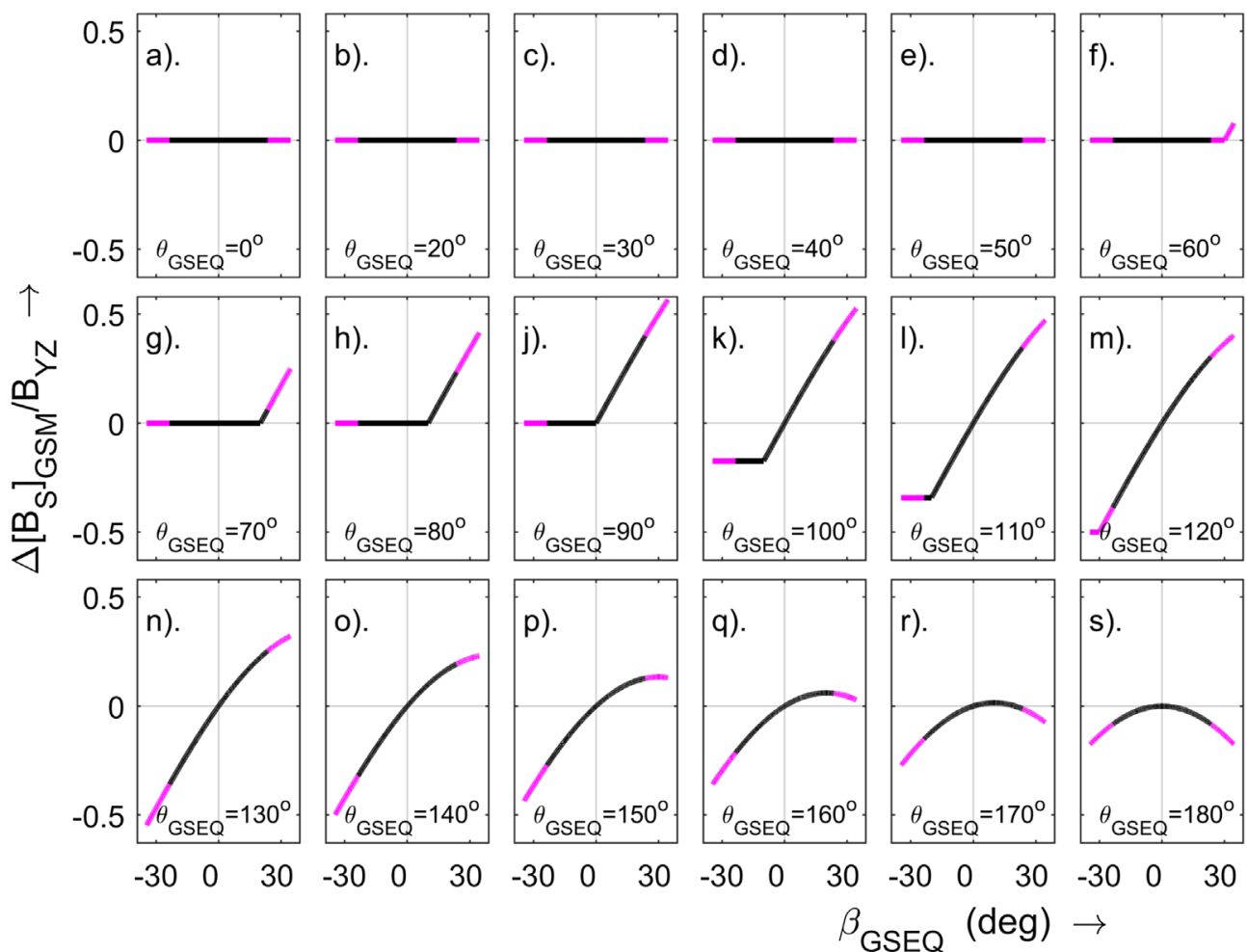

Figure B3. The same as Figure B2 for the $\Delta\left[B_{S}\right]_{\mathrm{GSM}} / B_{Y Z} \mathrm{IMF}$ orientation factor. Note that $\theta_{\mathrm{GSEQ}}=90^{\circ}$ is for IMF in the solar equatorial plane as used by Russell \& McPherron (1973) with this IMF orientation factor.

GSEQ frame) there is a reduction in geoeffectiveness at both positive and negative $\beta_{\mathrm{GSEQ}}$. Hence for events of large southward IMF in the GSEQ frame (such as ahead or in passing coronal mass ejections or co-rotating interaction regions) the $\mathrm{R}-\mathrm{M}$ effect reduces rather than enhances geoeffectiveness.

For smaller values of $\theta_{\mathrm{GSEQ}}$, the behaviour of the two coupling functions shows some differences. For the top row of Figure B3 (panels a-e) shows that for $\left[B_{S}\right]_{\mathrm{GSM}} / B_{Y Z}$ the $\mathrm{R}-\mathrm{M}$ mechanism has almost no effect because the coupling is always zero for northward IMF. On the other hand, the slight increase in $\sin ^{4}\left(\theta_{\mathrm{GSM}} / 2\right)$ with $\theta_{\mathrm{GSM}}$, even for these northward IMF conditions means that the R-M effect slightly increases geoeffectiveness for positive $\beta_{\mathrm{GSEQ}}$.

Panel (j) of Figure B3 describes the model used in the original paper by Russell \& McPherron (1973) with the IMF in the solar equatorial plane $\left(\theta_{\mathrm{GSEQ}}=90^{\circ}\right)$ and a half-wave rectifying coupling function. In this case we see clear enhancements when $\beta_{\mathrm{GSEQ}}>0$ and no decrease when $\beta_{\mathrm{GSEQ}}<0$. The equivalent plot for $\sin ^{4}\left(\theta_{\mathrm{GSM}} / 2\right)$, Figure B2 (j), shows the same sort of behavior but there is a decrease in geoeffectiveness when when $\beta_{\mathrm{GSEQ}}<0$; however, it is smaller than the gain for $\beta_{\mathrm{GSEQ}}>0$ and so there is still a net increase due to the $\mathrm{R}-\mathrm{M}$ effect.

Panel $(\mathrm{m})$ of both Figures B2 and B3 is significant $\left(\theta_{\mathrm{GSEQ}}=\right.$ $120^{\circ}$ ) because this is close to the point where the gain due to the R-M effect for $\beta_{\mathrm{GSEQ}}>0$ is very similar to the loss for $\beta_{\mathrm{GSEQ}}<0$. Hence this marks the point where on increasing $\theta_{\mathrm{GSEQ}}$, the R-M effect turns from a net enhancer of geoeffectiveness to a net reducer of it.

Cite this article as: Lockwood M, Owens MJ, Barnard LA, Haines C, Scott CJ, et al. 2020. Semi-annual, annual and Universal Time variations in the magnetosphere and in geomagnetic activity: 1. Geomagnetic data. J. Space Weather Space Clim. 10, 23. 